\title{
New Wilson-like theorems arising from Dickson polynomials
}

\author{
Antonia W. Bluher \\ National Security Agency \\ tbluher@access4less.net
}

\begin{abstract}
Wilson's Theorem states that the product of all nonzero elements of a finite field $\mathbb{F}_{q}$ is -1 . In this article, we define some natural subsets $S \subset \mathbb{F}_{q}^{\times}$ ( $q$ odd) and find formulas for the product of the elements of $S$, denoted $\prod S$. These new formulas are appealing for the simple, natural description of the sets $S$, and for the simplicity of the product. An example is $\prod\left\{a \in \mathbb{F}_{q}^{\times}\right.$: $1-a$ and $3+a$ are nonsquares $\}=2$ if $q \equiv \pm 1(\bmod 12)$, or -1 otherwise.
\end{abstract}

\section{Introduction}

John Wilson (1741-1793) stated that if $p$ is prime, then $p$ divides $(p-1) !+1$, i.e., the product of the nonzero elements of the finite field $\mathbb{F}_{p}$ is -1 . Wilson's Theorem is normally stated for fields of prime order, but its proof easily carries over to arbitrary finite fields $\mathbb{F}_{q}$, where $q=p^{n}$. One simply pairs each element of $\mathbb{F}_{q}^{\times}$with its multiplicative inverse, noting that 1 and -1 are the only elements that are equal to their own inverse. Each pair $\left\{a, a^{-1}\right\}$ multiplies to 1 , leaving us with $\prod \mathbb{F}_{q}^{\times}=1 \times-1=-1$.

Oystein Ore [7] provides the following history of Wilson's Theorem.

In the Meditationes Algebraicae by Edward Waring, published in Cambridge in 1770 , one finds [...] several announcements on the theory of numbers. One of them is the following: For any prime $p$ the quotient

$$
\frac{1 \cdot 2 \cdots \cdots(p-1)+1}{p}
$$

is an integer.

This result Waring ascribes to one of his pupils John Wilson (17411793). Wilson was a senior wrangler at Cambridge and left the field of mathematics quite early to study law. Later he became a judge and was knighted. Waring gives no proof of Wilson's theorem until the third edition of his Meditationes, which appeared in 1782. Wilson probably arrived at the result through numerical computations. Among the posthumous papers of Leibniz there were later found similar calculations on the remainders of $n$ !, and he seems to have made the same conjecture. The first proof of the theorem of Wilson was given by J. L. Lagrange in a treatise that appeared in 1770 . 
In [1, while investigating permutation properties of Dickson polynomials, we discovered the following "Wilson-like" theorems:

$$
\prod\left\{a \in \mathbb{F}_{q}^{\times}: a \text { and } 4-a \text { are nonsquares }\right\}=2
$$

and

$$
\prod\left\{a \in \mathbb{F}_{q}^{\times}:-a \text { and } 4+a \text { are nonsquares }\right\}=\left(\frac{2}{q}\right) \cdot 2,
$$

where $q$ is an odd prime power and $\left(\frac{a}{q}\right)$ denotes the Legendre symbol, defined for $a \in \mathbb{F}_{q}$ by

$$
\left(\frac{a}{q}\right)= \begin{cases}1 & \text { if } a \text { is a nonzero square in } \mathbb{F}_{q} \\ -1 & \text { if } a \text { is a nonsquare in } \mathbb{F}_{q}^{\times} \\ 0 & \text { if } a=0 .\end{cases}
$$

This article finds more formulas of this type, and again Dickson polynomials are a key ingredient. See [2] for a short exposition on these topics.

Define the following sets:

$$
\begin{gathered}
\mathcal{S}_{k}^{+}=\left\{a \in \mathbb{F}_{q}^{\times}:\left(\frac{a+k}{q}\right)=1\right\}, \quad \mathcal{S}_{k}^{-}=\left\{a \in \mathbb{F}_{q}^{\times}:\left(\frac{a+k}{q}\right)=-1\right\}, \\
\mathcal{S}_{k, \ell}^{++}=\mathcal{S}_{\ell, k}^{++}=\left\{a \in \mathbb{F}_{q}^{\times}:\left(\frac{a+k}{q}\right)=1,\left(\frac{a+\ell}{q}\right)=1\right\}, \\
\mathcal{S}_{k, \ell}^{+-}=\mathcal{S}_{\ell, k}^{-+}=\left\{a \in \mathbb{F}_{q}^{\times}:\left(\frac{a+k}{q}\right)=1,\left(\frac{a+\ell}{q}\right)=-1\right\}, \\
\mathcal{S}_{k, \ell}^{--}=\mathcal{S}_{\ell, k}^{--}=\left\{a \in \mathbb{F}_{q}^{\times}:\left(\frac{a+k}{q}\right)=-1,\left(\frac{a+\ell}{q}\right)=-1\right\},
\end{gathered}
$$

where $k, \ell \in \mathbb{F}_{q}$ and $k \neq \ell$. These definitions implicitly depend on $q$, which is always assumed to be an odd prime power. This article finds simple closed formulas for $\prod \mathcal{S}_{k}^{+}$ and $\prod \mathcal{S}_{k}^{-}$(Theorem 2.4) and $\prod \mathcal{S}_{k, \ell}^{ \pm, \pm}$(Section 7) for all $k$ and $\ell(k \neq \ell)$.

Define a related set $\mathcal{T}_{j, \ell}^{\varepsilon_{1}, \varepsilon_{2}}$ for $j, \ell \in \mathbb{F}_{q}, \varepsilon_{1}, \varepsilon_{2} \in\{+1,-1\}, j+\ell \neq 0$, as follows:

$$
\mathcal{T}_{j, \ell}^{\varepsilon_{1}, \varepsilon_{2}}=\left\{a \in \mathbb{F}_{q}^{\times}:\left(\frac{j-a}{q}\right)=\varepsilon_{1},\left(\frac{\ell+a}{q}\right)=\varepsilon_{2}\right\} .
$$

Note that

$$
\mathcal{T}_{j, \ell}^{\varepsilon_{1}, \varepsilon_{2}}=\mathcal{S}_{-j, \ell}^{\varepsilon \varepsilon_{1}, \varepsilon_{2}}, \quad \text { where } \varepsilon=\left(\frac{-1}{q}\right) .
$$

Our reason for introducing these sets is that $\prod \mathcal{S}_{k, \ell}^{ \pm, \pm}$requires separate formulas for the cases $q \equiv 1(\bmod 4)$ and $q \equiv 3(\bmod 4) ;$ whereas $\prod \mathcal{T}_{j, \ell}^{ \pm, \pm}$can be expressed with a single formula. For example, we will show that $\prod \mathcal{S}_{-4,0}^{--}=2$ if $q \equiv 1(\bmod 4)$, or $-1 / 2$ if $q \equiv 3(\bmod 4)$; whereas $\prod \mathcal{T}_{4,0}^{--}=2$ for all odd $q$. Some sample formulas that we will prove are the following:

$$
\prod \mathcal{T}_{2,2}^{-+}=(-1)^{\lfloor(q+3) / 8\rfloor}
$$




$$
\prod \mathcal{T}_{1,3}^{--}= \begin{cases}2 & \text { if } q \equiv \pm 1 \quad(\bmod 12) \\ -1 & \text { otherwise }\end{cases}
$$

If $j$ and $4-j$ are nonzero squares in $\mathbb{F}_{q}$, then we show in Theorem $7.1(i v)$ that

$$
\prod \mathcal{T}_{j, 4-j}^{--}=\left(\frac{2+\sqrt{j}}{q}\right) \cdot 2=\left(\frac{4+2 \sqrt{4-j}}{q}\right) \cdot 2=\left(\frac{2}{q}\right) \prod \mathcal{T}_{4-j, j}^{--}
$$

Here, $2 \pm \sqrt{j}$ are both squares or both nonsquares since their product $4-j$ is assumed to be a square, so the Legendre symbol is well defined, and similarly for $4 \pm 2 \sqrt{4-j}$. We show in Theorem 7.2 and Table 4 that

If $j$ is a square and $4-j$ is not, then $\prod \mathcal{T}_{j, 4-j}^{--}$is a square root of $j$.

If $4-j$ is a square and $j$ is not, then $\prod \mathcal{T}_{j, 4-j}^{--}$is a square root of $4-j$.

If $j$ and $4-j$ are nonsquares, then $\prod \mathcal{T}_{j, 4-j}^{+-}$is a square root of $j /(4-j)$.

In each case, a prescription is given that determines which square root to select. Thus, each formula is determined completely, and not just up to sign. In this article we find formulas for all $\prod \mathcal{T}_{j, \ell}^{\varepsilon_{1}, \varepsilon_{2}}(j \neq-\ell)$. See Tables 2 and 4 for a complete list when $j+\ell=4$. For arbitrary $j, \ell$ with $j+\ell \neq 0, \prod \mathcal{T}_{j, \ell}^{\varepsilon_{1}, \varepsilon_{2}}$ can be determined with our rescaling formula, given in Section 5 .

As hinted by (7), the above results are related to rational reciprocity. For example, consider the case $j=\ell=2$. As is well known (and will be proved in Lemma 4.3), 2 is a square in $\mathbb{F}_{q}$ iff $q= \pm 1(\bmod 8)$. Assuming $\left(\frac{2}{q}\right)=1$, (17) implies that $\prod \mathcal{T}_{2,2}^{--}=$ $\left(\frac{2+\sqrt{2}}{q}\right) \cdot 2$. On the other hand, $\prod \mathcal{T}_{2,2}^{--}=(-1)^{(q-\varepsilon) / 8} \cdot 2$ by Theorem $7.1(i i)$, where $\varepsilon=\left(\frac{-1}{q}\right)$. This equals 2 if $q \equiv \pm 1(\bmod 16)$ or -2 if $q \equiv 8 \pm 1(\bmod 16)$. The conclusion is that $2+\sqrt{2}$ is a square in $\mathbb{F}_{q}$ if $q \equiv \pm 1(\bmod 16)$ and a nonsquare if $q \equiv 8 \pm 1(\bmod 16)$. Although known (see [4]), it is nonetheless interesting that rational reciprocity arises in this context. For more examples of how our work relates to rational reciprocity, see Section 9 .

Of independent interest is our Theorem 4.1, which gives an explicit bijection between sets $\{v, 1 / v,-v,-1 / v\} \subset \mu_{2 q-2} \cup \mu_{2 q+2}$ and $\mathbb{F}_{q}$. The bijection is used to neatly characterize $\tau \in \mathbb{F}_{q}$ such that $\left(\frac{\tau}{q}\right)=\varepsilon_{1},\left(\frac{\tau+1}{q}\right)=\varepsilon_{2}$ for each pair $\left(\varepsilon_{1}, \varepsilon_{2}\right)$. Several applications of this result are given (see Section 4), for example a simple proof that $\left(\frac{2}{q}\right)=1$ iff $q \equiv \pm 1(\bmod 8)$ and $\left(\frac{-3}{q}\right)=1$ iff $q \equiv 1(\bmod 3)$.

Notation: Throughout, $q$ denotes a power of an odd prime, $\mathbb{F}_{q}$ the field with $q$ elements, $\mathbb{F}_{q}^{\times}$its group of nonzero elements under multiplication, and $\overline{\mathbb{F}}_{q}$ its algebraic closure. If $(d, q)=1$ then $\mu_{d}$ denotes the $d$-th roots of unity in $\overline{\mathbb{F}}_{q}$. If $c$ is a real number, then $\lfloor c\rfloor$ denotes the largest integer that is $\leq c$. If $S$ is a subset of $\mathbb{F}_{q}^{\times}$, then $\prod S$ denotes the product of the elements of $S$, and $|S|$ denotes its cardinality. By convention, $\prod \emptyset=1$. If $S$ and $S^{\prime}$ are disjoint, then $\prod S \times \prod S^{\prime}=\prod\left(S \cup S^{\prime}\right)$. We assume basic knowledge of 
finite fields, as can be found in [6, Chapter 2], and properties of the Legendre symbol over prime fields $\mathbb{F}_{p}$, as can be found in [8]. It is well known that $\mathbb{F}_{q}^{\times}$is cyclic. If $g$ is a generator, then $g^{i}$ is a square if and only if $i$ is even, and $g^{(q-1) / 2}=-1$. From this one can deduce the well-known properties of Legendre symbols that $\left(\frac{a}{q}\right)=a^{(q-1) / 2}$ and $\left(\frac{a}{q}\right)\left(\frac{b}{q}\right)=\left(\frac{a b}{q}\right)$ for all $a, b \in \mathbb{F}_{q}$. We often identify the symbols + and - with 1 and -1 , respectively. Thus, for $\varepsilon_{1}, \varepsilon_{2} \in\{+,-\}$ we have

$$
\begin{aligned}
& \mathcal{S}_{k, \ell}^{\varepsilon_{1}, \varepsilon_{2}}=\left\{a \in \mathbb{F}_{q}^{\times}:\left(\frac{a+k}{q}\right)=\varepsilon_{1}, \quad\left(\frac{a+\ell}{q}\right)=\varepsilon_{2}\right\}, \\
& \mathcal{T}_{j, \ell}^{\varepsilon_{1}, \varepsilon_{2}}=\left\{a \in \mathbb{F}_{q}^{\times}:\left(\frac{j-a}{q}\right)=\varepsilon_{1}, \quad\left(\frac{\ell+a}{q}\right)=\varepsilon_{2}\right\},
\end{aligned}
$$

where $j, k, \ell \in \mathbb{F}_{q}, j+\ell \neq 0$, and $k-\ell \neq 0$. We define sets that are similar to $\mathcal{S}_{k, \ell}^{\varepsilon_{1}, \varepsilon_{2}}$ but may contain 0 :

$$
\mathcal{A}_{k, \ell}^{\varepsilon_{1}, \varepsilon_{2}}=\left\{a \in \mathbb{F}_{q}:\left(\frac{a+k}{q}\right)=\varepsilon_{1}, \quad\left(\frac{a+\ell}{q}\right)=\varepsilon_{2}\right\} .
$$

Note that

$$
\mathcal{A}_{k, \ell}^{\varepsilon_{1}, \varepsilon_{2}}= \begin{cases}\mathcal{S}_{k, \ell}^{\varepsilon_{1}, \varepsilon_{2}} \sqcup\{0\} & \text { if }\left(\frac{k}{q}\right)=\varepsilon_{1} \text { and }\left(\frac{\ell}{q}\right)=\varepsilon_{2}, \\ \mathcal{S}_{k, \ell}^{\varepsilon_{1}, \varepsilon_{2}} & \text { otherwise, }\end{cases}
$$

where $\sqcup$ denotes the disjoint union, that is, the union of sets that are known to be pairwise disjoint. We sometimes abbreviate "if and only if" by "iff" or by the symbol $\Longleftrightarrow$. Because it arises often, we set

$$
\varepsilon=\left(\frac{-1}{q}\right)=(-1)^{(q-1) / 2} \in \mathbb{Z} .
$$

Starting in Section 8.1, we use the notation

$$
\langle u\rangle=u+1 / u .
$$

If $\lambda, \beta \in \mathbb{F}_{q}, \lambda \neq 0$, and $S \subset \mathbb{F}_{q}$, we define

$$
\lambda S+\beta=\{\lambda s+\beta: s \in S\} .
$$

One sees easily from the definitions that if $\nu=\left(\frac{\lambda}{q}\right)$ then for $\varepsilon_{1}, \varepsilon_{2} \in\{1,-1\}$,

$$
\begin{gathered}
\lambda \mathcal{A}_{k, \ell}^{\varepsilon_{1}, \varepsilon_{2}}+\beta=\mathcal{A}_{\lambda k-\beta, \lambda \ell-\beta}^{\nu \varepsilon_{1}, \nu \varepsilon_{2}}, \\
\mathcal{S}_{\lambda k}^{\varepsilon_{1}}=\lambda \mathcal{S}_{k}^{\varepsilon_{1} \nu}, \quad \mathcal{S}_{\lambda k, \lambda \ell}^{\varepsilon_{1}, \varepsilon_{2}}=\lambda \mathcal{S}_{k, \ell}^{\nu \varepsilon_{1}, \nu \varepsilon_{2}}, \quad \mathcal{T}_{\lambda j, \lambda \ell}^{\varepsilon_{1}, \varepsilon_{2}}=\lambda \mathcal{T}_{j, \ell}^{\nu \varepsilon_{1}, \nu \varepsilon_{2}} .
\end{gathered}
$$

This leads to "rescaling formulas":

$$
\prod \mathcal{S}_{\lambda k, \lambda \ell}^{\varepsilon_{1}, \varepsilon_{2}}=\lambda^{s} \prod \mathcal{S}_{k, \ell}^{\nu \varepsilon_{1}, \nu \varepsilon_{2}}, \quad \prod \mathcal{T}_{\lambda j, \lambda \ell}^{\varepsilon_{1}, \varepsilon_{2}}=\lambda^{t} \prod \mathcal{T}_{j, \ell}^{\nu \varepsilon_{1}, \nu \varepsilon_{2}},
$$

where $s$ is the cardinality of $\mathcal{S}_{k, \ell}^{\nu \varepsilon_{1}, \nu \varepsilon_{2}}$ and $t$ is the cardinality of $\mathcal{T}_{j, \ell}^{\nu \varepsilon_{1}, \nu \varepsilon_{2}}$. Here $s$ and $t$ will be computed in Section 4, resulting in our rescaling formula in Section 5. Using this, we can (and will) normalize by a scalar multiple to make $\ell-k=4$ and $\ell+j=4$.

The author thanks Art Drisko for thoroughly reviewing this article and providing many thoughtful comments. 


\section{Formulas for $\prod \mathcal{S}_{k}^{+}$and $\prod \mathcal{S}_{k}^{-}$}

This section gives simple formulas for $\prod \mathcal{S}_{k}^{+}$and $\prod \mathcal{S}_{k}^{-}$for all $k \in \mathbb{F}_{q}$. Lemma 2.1 is well known.

Lemma $2.1\left|\mathcal{S}_{0}^{+}\right|=\left|\mathcal{S}_{0}^{-}\right|=(q-1) / 2, \prod \mathcal{S}_{0}^{+}=(-1)^{(q+1) / 2}$, and $\prod \mathcal{S}_{0}^{-}=(-1)^{(q-1) / 2}$.

Proof. $\left|\mathcal{S}_{0}^{+}\right|=\left|\mathcal{S}_{0}^{-}\right|=(q-1) / 2$ because there are exactly $(q-1) / 2$ nonzero squares and the same number of nonsquares in $\mathbb{F}_{q}$. Let $g$ be a generator of $\mathbb{F}_{q}^{\times}$. Then $\mathcal{S}_{0}^{+}=$ $\left\{g^{2}, g^{4}, \ldots, g^{2 k}\right\}$ where $k=(q-1) / 2$. The product of these is $g^{s}$, where $s=2+4+$ $\ldots+2 k=k(k+1)$. Since $g^{k}=g^{(q-1) / 2}=-1, g^{s}=(-1)^{(q+1) / 2}$. Since $\mathcal{S}_{0}^{+} \sqcup \mathcal{S}_{0}^{-}=\mathbb{F}_{q}^{\times}$, $\prod \mathcal{S}_{0}^{-}=-1 / \prod \mathcal{S}_{0}^{+}=(-1)^{(q-1) / 2}$ by Wilson's Theorem.

Lemma $2.2\left|\mathcal{S}_{1}^{+}\right|=(q-3) / 2$ and $\prod \mathcal{S}_{1}^{+}=(-1)^{(q-1) / 2} / 2$.

Proof. Let $R$ be a complete set of representatives for $\mathbb{F}_{q}^{\times} /\{ \pm 1\}$ such that $1 \in R$. Now $\mathcal{S}_{1}^{+}=\left\{a \in \mathbb{F}_{q}^{\times}:\left(\frac{a+1}{q}\right)=1\right\}=\left\{s^{2}-1: s \in R, s \neq 1\right\}$, so $\left|\mathcal{S}_{1}^{+}\right|=|R|-1$ and

$$
\begin{aligned}
(-1)^{|R|-1} \prod \mathcal{S}_{1}^{+} & =\prod\{(1-s)(1+s): s \in R, s \neq 1\} \\
& =\prod\left\{1-t: t \in \mathbb{F}_{q}^{\times}, t \neq \pm 1\right\}=\prod \mathbb{F}_{q}^{\times} / 2=-1 / 2 .
\end{aligned}
$$

Since $|R|=(q-1) / 2$, the result follows.

Lemma $2.3\left|\mathcal{S}_{1}^{-}\right|=(q-1) / 2$ and $\prod \mathcal{S}_{1}^{-}=(-1)^{(q-1) / 2} \cdot 2$.

Proof. For each $a \in \mathbb{F}_{q}^{\times},\left(\frac{a+1}{q}\right)$ is 0,1 , or -1 ; furthermore, $\left(\frac{a+1}{q}\right)=0$ iff $a=-1$. Thus, $\mathbb{F}_{q}^{\times}=\mathcal{S}_{1}^{+} \sqcup \mathcal{S}_{1}^{-} \sqcup\{-1\}$. This shows that $\left|\mathcal{S}_{1}^{-}\right|=(q-1)-1-\left|\mathcal{S}_{1}^{+}\right|=q-2-(q-3) / 2=$ $(q-1) / 2$, and that $\prod \mathcal{S}_{1}^{+} \times \prod \mathcal{S}_{1}^{-} \times(-1)=\prod \mathbb{F}_{q}^{\times}=-1$, by Wilson's Theorem. Since $\prod \mathcal{S}_{1}^{+}=(-1)^{(q-1) / 2} / 2$, we find that $\prod \mathcal{S}_{1}^{-}=(-1)^{(q-1) / 2} \cdot 2$.

Theorem 2.4 Let $k \in \mathbb{F}_{q}$. Then

$$
\begin{aligned}
& \prod \mathcal{S}_{k}^{+}= \begin{cases}(-1)^{(q-1) / 2} /(2 k) & \text { if } k \text { is a nonzero square in } \mathbb{F}_{q} \\
(-1)^{(q+1) / 2} \cdot 2 & \text { if } k \text { is a nonsquare in } \mathbb{F}_{q} \\
(-1)^{(q+1) / 2} & \text { if } k=0 ;\end{cases} \\
& \prod \mathcal{S}_{k}^{-}= \begin{cases}(-1)^{(q-1) / 2} \cdot 2 & \text { if } k \text { is a nonzero square in } \mathbb{F}_{q} \\
(-1)^{(q+1) / 2} /(2 k) & \text { if } k \text { is a nonsquare in } \mathbb{F}_{q} \\
(-1)^{(q-1) / 2} & \text { if } k=0 .\end{cases}
\end{aligned}
$$

Proof. The result when $k=0$ is contained in Lemma 2.1, If $k \neq 0$, then let $\nu=\left(\frac{k}{q}\right)$. By (15) applied with $\lambda$ and $k$ replaced by $k$ and $1, \mathcal{S}_{k}^{\nu}=k \mathcal{S}_{1}^{+}$and $\mathcal{S}_{k}^{-\nu}=k \mathcal{S}_{1}^{-}$. Since $\left|\mathcal{S}_{1}^{+}\right|=(q-3) / 2,\left|\mathcal{S}_{1}^{-}\right|=(q-1) / 2$, and $k^{(q-1) / 2}=\nu$, we have

$$
\begin{aligned}
\prod \mathcal{S}_{k}^{-\nu} & =\prod\left(k \mathcal{S}_{1}^{-}\right)=k^{(q-1) / 2} \prod \mathcal{S}_{1}^{-}=\nu(-1)^{(q-1) / 2} \cdot 2, \\
\prod \mathcal{S}_{k}^{\nu} & =\prod\left(k \mathcal{S}_{1}^{+}\right)=k^{(q-3) / 2} \prod \mathcal{S}_{1}^{+}=(\nu / k)(-1)^{(q-1) / 2} / 2 .
\end{aligned}
$$

The result follows. 


\section{Relations between $\prod \mathcal{S}_{k, \ell}^{++}, \prod \mathcal{S}_{k, \ell}^{+-}, \prod \mathcal{S}_{k, \ell}^{-+}$, and $\prod \mathcal{S}_{k, \ell}^{--}$.}

In this section, we show that knowing one of $\prod \mathcal{S}_{k, \ell}^{++}, \prod \mathcal{S}_{k, \ell}^{+-}, \prod \mathcal{S}_{k, \ell}^{-+}$, and $\prod \mathcal{S}_{k, \ell}^{--}$ allows to easily compute the other three.

Proposition 3.1 Let $k, \ell$ be distinct elements of $\mathbb{F}_{q}$. Then

$$
\begin{gathered}
\prod \mathcal{S}_{k}^{+}= \begin{cases}\prod \mathcal{S}_{k, \ell}^{++} \times \prod \mathcal{S}_{k, \ell}^{+-} \times(-\ell) & \text { if }\left(\frac{k-\ell}{q}\right)=1 \text { and } \ell \neq 0 \\
\prod \mathcal{S}_{k, \ell}^{++} \times \prod \mathcal{S}_{k, \ell}^{+-} & \text {if }\left(\frac{k-\ell}{q}\right)=-1 \text { or } \ell=0 .\end{cases} \\
\prod \mathcal{S}_{\ell}^{-}= \begin{cases}\prod \mathcal{S}_{k, \ell}^{--} \times \prod \mathcal{S}_{k, \ell}^{+-} & \text {if }\left(\frac{\ell-k}{q}\right)=1 \text { or } k=0 \\
\prod \mathcal{S}_{k, \ell}^{--} \times \prod \mathcal{S}_{k, \ell}^{+-} \times(-k) & \text { if }\left(\frac{\ell-k}{q}\right)=-1 \text { and } k \neq 0 .\end{cases} \\
\prod \mathcal{S}_{k}^{-}= \begin{cases}\prod \mathcal{S}_{k, \ell}^{--} \times \prod \mathcal{S}_{k, \ell}^{-+} & \text {if }\left(\frac{k-\ell}{q}\right)=1 \text { or } \ell=0 \\
\prod \mathcal{S}_{k, \ell}^{--} \times \prod \mathcal{S}_{k, \ell}^{-+} \times(-\ell) & \text { if }\left(\frac{k-\ell}{q}\right)=-1 \text { and } \ell \neq 0 .\end{cases}
\end{gathered}
$$

Proof. $\mathcal{S}_{k}^{+}$consists of all $a \in \mathbb{F}_{q}^{\times}$such that $a+k$ is a nonzero square in $\mathbb{F}_{q}$. If $a \in \mathcal{S}_{k}^{+}$, then either $a+\ell=0, a+\ell$ is a nonzero square, or $a+\ell$ is a nonsquare in $\mathbb{F}_{q}$. The case $a+\ell=0$ can occur iff $-\ell \in \mathcal{S}_{k}^{+}$, i.e., iff $\left(\frac{k-\ell}{q}\right)=1$ and $-\ell \in \mathbb{F}_{q}^{\times}$. Thus,

$$
\mathcal{S}_{k}^{+}= \begin{cases}\mathcal{S}_{k \ell}^{++} \sqcup \mathcal{S}_{k \ell}^{+-} \sqcup\{-\ell\}, & \text { if }\left(\frac{k-\ell}{q}\right)=1 \text { and } \ell \neq 0, \\ \mathcal{S}_{k \ell}^{++} \sqcup \mathcal{S}_{k \ell}^{+-} & \text {if }\left(\frac{k-\ell}{q}\right)=-1 \text { or } \ell=0 .\end{cases}
$$

This proves the first formula, and the second is proved similarly, using

$$
\mathcal{S}_{\ell}^{-}= \begin{cases}\mathcal{S}_{k \ell}^{--} \sqcup \mathcal{S}_{k \ell}^{+-} \sqcup\{-k\}, & \text { if }\left(\frac{\ell-k}{q}\right)=-1 \text { and } k \neq 0, \\ \mathcal{S}_{k \ell}^{--} \sqcup \mathcal{S}_{k \ell}^{+-} & \text {if }\left(\frac{\ell-k}{q}\right)=1 \text { or } k=0 .\end{cases}
$$

The third formula is obtained by exchanging $k$ and $\ell$ in the second formula.

Knowing one of $\prod \mathcal{S}_{k, \ell}^{++}, \prod \mathcal{S}_{k, \ell}^{+-}, \prod \mathcal{S}_{\ell, k}^{+-}$, and $\prod \mathcal{S}_{k, \ell}^{--}$allows to easily compute the other three. For example, suppose $\prod \mathcal{S}_{k, \ell}^{++}$is known. Since $\prod \mathcal{S}_{k}^{+}$is known (by Theorem 2.4), (17) gives a formula for $\prod \mathcal{S}_{k, \ell}^{+-}$in terms of known quantities. Next, (18) can be used to solve for $\prod \mathcal{S}_{k, \ell}^{--}$, and finally (19) can be used to solve for $\prod \mathcal{S}_{k, \ell}^{-+}$.

\section{A correspondence between $\mu_{2 q+2} \cup \mu_{2 q-2}$ and $\mathbb{F}_{q}$}

The main result of this section is a correspondence $\tau=(1 / 4)(v-1 / v)^{2}$ between elements $v \in \mu_{2 q-2} \cup \mu_{2 q+2}$ and elements $\tau \in \mathbb{F}_{q}$, where $\mu_{d}$ denotes the set of $d$ th roots of unity in $\overline{\mathbb{F}}_{q}$. As will be shown in Theorem 4.1, this correspondence determines an explicit bijection between sets $\{v, 1 / v,-v,-1 / v\} \subset \mu_{2 q-2} \cup \mu_{2 q+2}$ and $\mathbb{F}_{q}$, and the multiplicative order of $v$ determines the square classes of $\tau$ and $\tau+1$. We apply this to easily compute the cardinalities of $\mathcal{A}_{k, \ell}^{ \pm, \pm}, \mathcal{S}_{k, \ell}^{ \pm, \pm}$, and $\mathcal{T}_{j, \ell}^{ \pm, \pm}$. Another application is a 
simple proof of the formulas for $\left(\frac{2}{q}\right)$ and $\left(\frac{-3}{q}\right)$. We remark that the cardinalities of $\mathcal{A}_{k, \ell}^{ \pm, \pm}$are well known in the literature and the computation is based on Jacobi sums; see [3] for a brief history. Our computation of $\left|\mathcal{A}_{k, \ell}^{ \pm, \pm}\right|$is very different from those in the literature. The cardinalities of $\mathcal{S}_{k, \ell}^{ \pm, \pm}$and $\mathcal{T}_{j, \ell}^{ \pm, \pm}$are needed to prove our rescaling formula and to obtain a relation between $\prod \mathcal{T}_{j, \ell}^{ \pm, \pm}$and $\prod \mathcal{T}_{\ell, j}^{ \pm, \pm}$. (See Section [5.)

If $d$ is even, then $v \in \mu_{d}$ implies that $\{v, 1 / v,-v,-1 / v\} \subset \mu_{d}$, and we call this an orbit. The orbit has exactly four elements unless $v^{4}=1$, in which case it has exactly two elements. Note that if $v^{\prime}$ is any element of the orbit, then $\left\{ \pm v^{\prime}, \pm 1 / v^{\prime}\right\}=\{ \pm v, \pm 1 / v\}$. The choice of which one to call $v$ is arbitrary.

Theorem 4.1 The orbits $\{v, 1 / v,-v,-1 / v\}$ of $\mu_{2(q-1)} \cup \mu_{2(q+1)}$ correspond one-to-one with $\tau \in \mathbb{F}_{q}$. In this correspondence, $\{v, 1 / v,-v,-1 / v\}$ is sent to $\tau=(1 / 4)(v-1 / v)^{2}$, and $\tau$ is sent to the orbit $\{ \pm \sqrt{\tau+1} \pm \sqrt{\tau}\}$. Further, $v^{4}=1 \Longleftrightarrow \tau \in\{-1,0\}$. If $\tau \notin\{-1,0\}$ (and consequently, $v^{4} \neq 1$ ), then for each pair $a, b \in\{ \pm 1\} \times\{ \pm 1\}$, we have

$$
\left(\frac{\tau}{q}\right)=a \text { and }\left(\frac{\tau+1}{q}\right)=b \Longleftrightarrow v^{q-a b}=b .
$$

Proof. The theorem claims a bijection between orbits $\{ \pm v, \pm 1 / v\} \subset \mu_{2 q-2} \cup \mu_{2 q+2}$ and elements of $\tau \in \mathbb{F}_{q}$, wherein $\{ \pm v, \pm 1 / v\}$ corresponds to $\tau=(1 / 4)(v-1 / v)^{2}$, and $\tau$ corresponds to the orbit $\{ \pm \sqrt{1+\tau} \pm \sqrt{\tau}\}$. Several things need to be demonstrated:

(a) If $\tau \in \mathbb{F}_{q}$, then $\{ \pm \sqrt{1+\tau} \pm \sqrt{\tau}\}$ really is an orbit;

(b) If $\{ \pm v, \pm 1 / v\}$ is an orbit, then $\tau=(1 / 4)(v-1 / v)^{2}$ is in $\mathbb{F}_{q}$ and is independent of the choice of $v$ within the orbit;

(c) The composition $\tau \mapsto\{ \pm \sqrt{\tau+1} \pm \sqrt{\tau}\}$ with $\{ \pm v, \pm 1 / v\} \mapsto(1 / 4)(v-1 / v)^{2}$ is the identity map on $\mathbb{F}_{q}$.

(d) The composition $\{ \pm v, \pm 1 / v\} \mapsto(1 / 4)(v-1 / v)^{2}$ with $\tau \mapsto\{ \pm \sqrt{\tau+1} \pm \sqrt{\tau}\}$ is the identity map on the set of orbits.

(a) Let $\tau \in \mathbb{F}_{q}$. For fixed choices of square roots, let $v=\sqrt{\tau+1}+\sqrt{\tau}$. Then $1 / v=\sqrt{\tau+1}-\sqrt{\tau}$, because

$$
(\sqrt{\tau+1}+\sqrt{\tau})(\sqrt{\tau+1}-\sqrt{\tau})=(\tau+1)-\tau=1 .
$$

Thus, $\{ \pm \sqrt{\tau+1} \pm \sqrt{\tau}\}=\{ \pm v, \pm 1 / v\}$. Since $(\sqrt{a})^{q} \in\{\sqrt{a},-\sqrt{a}\}$ when $a \in \mathbb{F}_{q}$, we have $v^{q} \in\{ \pm \sqrt{\tau+1} \pm \sqrt{\tau}\}=\{v, 1 / v,-v,-1 / v\}$. Then $v^{2 q} \in\left\{v^{2}, 1 / v^{2}\right\}$, whence $v \in \mu_{2 q-2} \cup \mu_{2 q+2}$.

(b) Let $\{ \pm v, \pm 1 / v\}$ be an orbit. It is clear that $(1 / 4)(v-1 / v)^{2}$ is invariant under $v \mapsto-v$ and $v \mapsto 1 / v$, so $\tau=(1 / 4)(v-1 / v)^{2}$ is a well-defined function of the orbit (independent of which one is called $v$ ). Since $v^{2 q} \in\left\{v^{2}, v^{-2}\right\}$, we have $v^{q} \in\{ \pm v, \pm 1 / v\}$. Thus, $\tau=(1 / 4)\left(v^{q}-1 / v^{q}\right)^{2}=\tau^{q}$, showing that $\tau \in \mathbb{F}_{q}$.

(c) We show that if $\tau \in \mathbb{F}_{q}$ and $v=\sqrt{\tau+1}+\sqrt{\tau}$ then $\tau=(1 / 4)(v-1 / v)^{2}$. Indeed, $v-1 / v=(\sqrt{\tau+1}+\sqrt{\tau})-(\sqrt{\tau+1}-\sqrt{\tau})=2 \sqrt{\tau}$, so $\tau=(1 / 4)(v-1 / v)^{2}$. 
(d) Given $v \in \mu_{2(q+1)} \cup \mu_{2(q-1)}$, let $\tau=(1 / 4)(v-1 / v)^{2}$, and we claim $v \in\{ \pm \sqrt{\tau+1} \pm$ $\sqrt{\tau}\}$. Since $\tau+1=(1 / 4)(v-1 / v)^{2}+1=(1 / 4)(v+1 / v)^{2}$, we see that $(v-1 / v) / 2=\sqrt{\tau}$, $(v+1 / v) / 2=\sqrt{\tau+1}$ for the right choices of square root, therefore $v=(v+1 / v) / 2+$ $(v-1 / v) / 2=\sqrt{\tau+1}+\sqrt{\tau}$.

Now we prove $v^{4}=1 \Longleftrightarrow \tau \in\{0,-1\}$. The orbits containing a fourth root of unity are $\{1,-1\}$ and $\{i,-i\}$, where $\pm i$ are the square roots of -1 . These correspond to $\tau=(1 / 4)(v-1 / v)^{2} \in\{0,-1\}$.

Finally, we show the claimed relations between $\left(\frac{\tau}{q}\right),\left(\frac{\tau+1}{q}\right)$, and $v$. If $\tau$ and $\tau+1$ are squares then $v \in \mathbb{F}_{q}$, so $v^{q-1}=1$. If $\tau+1$ is a square and $\tau$ is a nonsquare, then $v^{q}=\sqrt{\tau+1}-\sqrt{\tau}=1 / v$, so $v^{q+1}=1$. If $\tau+1$ is a nonsquare and $\tau$ is a square, then $v^{q}=-(\sqrt{\tau+1}-\sqrt{\tau})=-1 / v$, so $v^{q+1}=-1$. If $\tau$ and $\tau+1$ are nonsquares, then $v^{q}=-v$, so $v^{q-1}=-1$.

Note that we can write $\mathbb{F}_{q}$ as a disjoint union:

$$
\mathbb{F}_{q}=\{0\} \sqcup\{-1\} \sqcup \mathcal{A}_{0,1}^{++} \sqcup \mathcal{A}_{0,1}^{+-} \sqcup \mathcal{A}_{0,1}^{-+} \sqcup \mathcal{A}_{0,1}^{--} .
$$

In the correspondence, $\tau=0$ corresponds to the orbit $\{1,-1\}, \tau=-1$ corresponds to the orbit $\{ \pm \sqrt{-1}\}$, and $\tau \in \mathcal{A}_{0,1}^{\varepsilon_{1}, \varepsilon_{2}}$ corresponds to all orbits $\{ \pm v, \pm 1 / v\}$ such that $v^{q-\varepsilon_{1} \varepsilon_{2}}=\varepsilon_{2}$ and $v^{4} \neq 1$. In other words,

$$
\mathcal{A}_{0,1}^{\varepsilon_{1}, \varepsilon_{2}}=\left\{(v-1 / v)^{2} / 4: v^{q-\varepsilon_{1} \varepsilon_{2}}=\varepsilon_{2}, v^{4} \neq 1\right\} .
$$

Corollary 4.2 For all $\varepsilon_{1}, \varepsilon_{2} \in\{ \pm 1\}$,

$$
\mathcal{A}_{-2,2}^{\varepsilon_{1}, \varepsilon_{2}}=\left\{v^{2}+v^{-2}: v^{q-\varepsilon_{1} \varepsilon_{2}}=\varepsilon_{2} \text { and } v^{4} \neq 1\right\} .
$$

Proof. Consider what happens to the equality (24) when $\tau$ is mapped to $4 \tau+2$. First, $\tau \in \mathcal{A}_{0,1}^{\varepsilon_{1}, \varepsilon_{2}} \Longleftrightarrow 4 \tau+2 \in \mathcal{A}_{-2,2}^{\varepsilon_{1}, \varepsilon_{2}}$. Second, $\tau=(v-1 / v)^{2} / 4 \Longleftrightarrow 4 \tau+2=v^{2}+v^{-2}$. The result follows.

We give several applications of Theorem 4.1, beginning with a simple proof of the formulas for $\left(\frac{2}{q}\right)$ and $\left(\frac{-3}{q}\right)$. Lemma 4.3 is well known.

Lemma 4.3 Let $q=p^{n}$, where $p$ is an odd prime. Then -1 is a square in $\mathbb{F}_{q}$ if and only if $q \equiv 1(\bmod 4), 2$ is a square in $\mathbb{F}_{q}$ if and only if $q \equiv \pm 1(\bmod 8)$, and -3 is a nonzero square in $\mathbb{F}_{q}$ if and only if $q \equiv 1(\bmod 3)$. If $a \in \mathbb{F}_{p}$ then $\left(\frac{a}{q}\right)=\left(\frac{a}{p}\right)^{n}$. If $\varepsilon=(-1)^{(q-1) / 2}$, then $(q-\varepsilon) / 4$ is an integer and

$$
\left(\frac{2}{q}\right)=(-1)^{(q-\varepsilon) / 4}
$$

Proof. For the first assertion, $\left(\frac{-1}{q}\right)=(-1)^{(q-1) / 2}$ and $(q-1) / 2$ is even iff $q \equiv 1$ $(\bmod 4)$. For the penultimate assertion, if $a \in \mathbb{F}_{p}^{\times}$, then

$$
\left(\frac{a}{q}\right)=a^{(q-1) / 2}=\left(a^{(p-1) / 2}\right)^{m}=\left(\frac{a}{p}\right)^{m},
$$


where $m=\left(p^{n}-1\right) /(p-1)=1+p+\cdots+p^{n-1}$. Since $m \equiv n(\bmod 2)$, we have $\left(\frac{a}{q}\right)=\left(\frac{a}{p}\right)^{n}$.

Now we prove that -3 is a square in $\mathbb{F}_{q} \Longleftrightarrow q \equiv 1(\bmod 3)$. Set $v=\omega$, where $\omega$ is a primitive cube root of unity, and let $\tau \in \mathbb{F}_{q}$ correspond to the orbit of $v$. Then $\tau=(1 / 4)(v-1 / v)^{2}=(1 / 4)\left(\omega^{2}+\omega^{-2}-2\right)=(1 / 4)\left(\omega^{2}+\omega-2\right)=-3 / 4$, and $\tau+1=1 / 4$. By Theorem 4.1,

$$
\left(\frac{-3}{q}\right)=1 \Longleftrightarrow \tau \text { and } \tau+1 \text { are squares } \Longleftrightarrow \omega^{q-1}=1
$$

and the latter is equivalent to $3 \mid q-1$.

Next, we prove that 2 is a square in $\mathbb{F}_{q} \Longleftrightarrow q \equiv \pm 1(\bmod 8)$. Let $v=\zeta$, a primitive eighth root of unity, and let $\tau \in \mathbb{F}_{q}$ correspond to the orbit of $v$. Then $\tau=(1 / 4)(\zeta-1 / \zeta)^{2}=(1 / 4)(i+1 / i-2)=-2 / 4$ and $\tau+1=2 / 4$, where $i=\zeta^{2}$. By Theorem 4.1,

$$
\begin{gathered}
\left(\frac{-2}{q}\right)=\left(\frac{2}{q}\right)=1 \Longleftrightarrow \zeta^{q-1}=1 \Longleftrightarrow 8 \mid(q-1), \\
\left(\frac{-2}{q}\right)=-1,\left(\frac{2}{q}\right)=1 \Longleftrightarrow \zeta^{q+1}=1 \Longleftrightarrow 8 \mid(q+1) .
\end{gathered}
$$

Thus, $\left(\frac{2}{q}\right)=1$ iff $q \equiv \pm 1(\bmod 8)$.

Finally, we prove (26) $)$. Note that $q \equiv \varepsilon(\bmod 4)$. Since $(q-\varepsilon) / 4$ is even iff $q \equiv \varepsilon$ $(\bmod 8)$ iff $q \equiv \pm 1(\bmod 8)$ iff $\left(\frac{2}{q}\right)=1$, we see that $\left(\frac{2}{q}\right)=(-1)^{(q-\varepsilon) / 4}$.

A second application of Theorem 4.1 is the following counting result. Again, the result is known, but the method of proof is new.

Proposition 4.4 Let $\mathcal{A}_{k, \ell}^{ \pm, \pm}$be as in (11)). Then $\left|\mathcal{A}_{0,1}^{++}\right|=\lfloor(q-3) / 4\rfloor,\left|\mathcal{A}_{0,1}^{+-}\right|=\lfloor(q+$ $1) / 4\rfloor,\left|\mathcal{A}_{0,1}^{-+}\right|=\lfloor(q-1) / 4\rfloor,\left|\mathcal{A}_{0,1}^{--}\right|=\lfloor(q-1) / 4\rfloor$.

Proof. The theorem shows that $v \mapsto \tau=(1 / 4)(v-1 / v)^{2}$ gives a four-to-one map from $\left(\mu_{2(q+1)} \cup \mu_{2(q-1)}\right) \backslash \mu_{4}$ onto $\mathbb{F}_{q} \backslash\{0,-1\}$, and that

$$
\tau \in \mathcal{A}_{0,1}^{\varepsilon_{1}, \varepsilon_{2}} \Longleftrightarrow v^{q-\varepsilon_{1} \varepsilon_{2}}=\varepsilon_{2} \text { and } v^{4} \neq 1
$$

Each set of $v$ 's in (27) can be described as $S_{a, b} \backslash \mu_{4}$, where $S_{a, b}=\left\{v \in \overline{\mathbb{F}}_{q}: v^{q+a}=b\right\}$ and $a, b \in\{1,-1\}$. Note that $\left|S_{a, b}\right|=q+a$. Also, the cardinality of $S_{a, b} \backslash \mu_{4}$ is divisible by 4 , since it consists of disjoint orbits of size 4 . Let $i=\left|S_{a, b} \cap \mu_{4}\right|$. Then $0 \leq i \leq 4$, and $\left|S_{a, b}\right|-i=q+a-i$ is divisible by 4 . Since $q+a$ is even, $i$ is even. That is, $i \in\{0,2,4\}$. If $b=1$ then $1 \in S_{a, b} \cap \mu_{4}$, so $i \in\{2,4\}$ and $\left|S_{a, b} \backslash \mu_{4}\right| / 4 \in\{(q+a-2) / 4,(q+a-4) / 4\}$. This number must be an integer, so $\left|S_{a, b} \backslash \mu_{4}\right| / 4=\lfloor(q+a-2) / 4\rfloor$. Thus, $\left|\mathcal{A}_{0,1}^{++}\right|=\lfloor(q-3) / 4\rfloor$ and $\left|\mathcal{A}_{0,1}^{-+}\right|=\lfloor(q-1) / 4\rfloor$. If $b=-1$, then $1 \notin S_{a, b}$, so $i \in\{0,2\}$. Then $\left|S_{a, b} \backslash \mu_{4}\right| / 4 \in$ $\{(q+a) / 4,(q+a-2) / 4\}$. Again, this must be an integer, so $\left|S_{a, b} \backslash \mu_{4}\right| / 4=\lfloor(q+a) / 4\rfloor$. Thus, $\left|\mathcal{A}_{0,1}^{+-}\right|=\lfloor(q+1) / 4\rfloor$ and $\left|\mathcal{A}_{0,1}^{--}\right|=\lfloor(q-1) / 4\rfloor$.

Corollary 4.5 If a is a random nonsquare in $\mathbb{F}_{q} \backslash\{0,-1\}$ (under the uniform distribution), then $a+1$ is a square with probability exactly $1 / 2$. 
Proof. The value $a$ is randomly selected from $\mathcal{A}_{0,1}^{-+} \sqcup \mathcal{A}_{0,1}^{--}$. The corollary follows by noting that $\left|\mathcal{A}_{0,1}^{-+}\right|=\left|\mathcal{A}_{0,1}^{--}\right|$.

Let $\varepsilon=\left(\frac{-1}{q}\right)$ and $m=(q-\varepsilon) / 4 \in \mathbb{Z}$. In Proposition 4.4, it is useful to note that $\lfloor(q+1) / 4\rfloor=m,\lfloor(q-3) / 4\rfloor=m-1$, and $\lfloor(q-1) / 4\rfloor=m+(\varepsilon-1) / 2$. Thus,

$$
\left|\mathcal{A}_{0,1}^{++}\right|=m-1, \quad\left|\mathcal{A}_{0,1}^{+-}\right|=m, \quad\left|\mathcal{A}_{0,1}^{-+}\right|=\left|\mathcal{A}_{0,1}^{--}\right|=m+(\varepsilon-1) / 2 .
$$

Proposition 4.6 Let $k, \ell$ be distinct elements of $\mathbb{F}_{q}$ and $\nu=\left(\frac{\ell-k}{q}\right)$. Then

$$
\left|\mathcal{A}_{k, \ell}^{\nu, \nu}\right|=m-1, \quad\left|\mathcal{A}_{k, \ell}^{\nu,-\nu}\right|=m, \quad\left|\mathcal{A}_{k, \ell}^{-\nu, \nu}\right|=\left|\mathcal{A}_{k, \ell}^{-\nu,-\nu}\right|=m+(\varepsilon-1) / 2,
$$

where $m=(q-\varepsilon) / 4$. Also, if $\mu \in\{1,-1\}$ then

$$
\left|\mathcal{A}_{k, \ell}^{\varepsilon \mu, \mu}\right|=m-\gamma, \quad \text { where } \gamma= \begin{cases}1 & \text { if } \mu=\nu \\ 0 & \text { if } \mu=-\nu .\end{cases}
$$

Proof. The result is true when $k=0$ and $\ell=1$ by (28). If $k, \ell$ are arbitrary distinct elements of $\mathbb{F}_{q}$, then $(\ell-k) \mathcal{A}_{0,1}^{\nu \varepsilon_{1}, \nu \varepsilon_{2}}-k=\mathcal{A}_{k, \ell}^{\varepsilon_{1}, \varepsilon_{2}}$ by (14). In particular, $\mathcal{A}_{k, \ell}^{\varepsilon_{1}, \varepsilon_{2}}$ and $\mathcal{A}_{0,1}^{\nu \varepsilon_{1}, \nu \varepsilon_{2}}$ have the same cardinality. The result follows.

Corollary 4.7 Let $j, k, \ell \in \mathbb{F}_{q}, k \neq \ell, j+\ell \neq 0$. Then

$$
\begin{aligned}
& \left|\mathcal{S}_{k, \ell}^{\varepsilon_{1}, \varepsilon_{2}}\right|= \begin{cases}\left|\mathcal{A}_{k, \ell}^{\varepsilon_{1}, \varepsilon_{2}}\right|-1 & \text { if }\left(\frac{k}{q}\right)=\varepsilon_{1} \text { and }\left(\frac{\ell}{q}\right)=\varepsilon_{2}, \\
\left|\mathcal{A}_{k, \ell}^{\varepsilon_{1}, \varepsilon_{2}}\right| & \text { otherwise; }\end{cases} \\
& \left|\mathcal{T}_{j, \ell}^{\varepsilon_{1}, \varepsilon_{2}}\right|= \begin{cases}\left|\mathcal{A}_{-j, \ell}^{\varepsilon \varepsilon_{1}, \varepsilon_{2}}\right|-1 & \text { if }\left(\frac{j}{q}\right)=\varepsilon_{1} \text { and }\left(\frac{\ell}{q}\right)=\varepsilon_{2}, \\
\left|\mathcal{A}_{-j, \ell}^{\varepsilon \varepsilon_{1}, \varepsilon_{2}}\right| & \text { otherwise. }\end{cases}
\end{aligned}
$$

Here $\left|\mathcal{A}_{k, \ell}^{\varepsilon_{1}, \varepsilon_{2}}\right|$ and $\left|\mathcal{A}_{-j, \ell}^{\varepsilon \varepsilon_{1}, \varepsilon_{2}}\right|$ can be determined using Proposition 4.6.

Proof. This follows from (12) and (4).

\section{Rescaling}

In Sections 7 and 8 we will use Dickson polynomials to find formulas for $\prod \mathcal{T}_{j, \ell}^{ \pm, \pm}$ when $j+\ell=4$. Lemma 5.1 allows us to extrapolate these formulas to arbitrary pairs $j^{\prime}$ and $\ell^{\prime}$ with $j^{\prime}+\ell^{\prime} \neq 0$. Similar comments apply to $\mathcal{S}_{k, \ell}^{ \pm, \pm}$.

Lemma 5.1 (Rescaling Formula) Let $j^{\prime}, \ell^{\prime}$ be arbitrary elements of $\mathbb{F}_{q}$ such that $j^{\prime}+\ell^{\prime} \neq$ 0 , and let $\varepsilon_{1}, \varepsilon_{2} \in\{1,-1\}$. Further, let $\lambda=\left(j^{\prime}+\ell^{\prime}\right) / 4, \nu=\left(\frac{j^{\prime}+\ell^{\prime}}{q}\right), j=j^{\prime} / \lambda$, and $\ell=\ell^{\prime} / \lambda$. (Note that $\left.j+\ell=4\right)$. Then

$$
\prod \mathcal{T}_{j^{\prime}, \ell^{\prime}}^{\varepsilon_{1}, \varepsilon_{2}}=\prod \mathcal{S}_{-j^{\prime}, \ell^{\prime}}^{\varepsilon \varepsilon_{1}, \varepsilon_{2}}=\lambda^{m-\beta-\gamma} \prod \mathcal{S}_{-j, \ell}^{\nu \varepsilon \varepsilon_{1}, \nu \varepsilon_{2}}=\lambda^{m-\beta-\gamma} \prod \mathcal{T}_{j, \ell}^{\nu \varepsilon_{1}, \nu \varepsilon_{2}},
$$


where $\varepsilon=\left(\frac{-1}{q}\right), m=(q-\varepsilon) / 4$,

$$
\begin{gathered}
\beta= \begin{cases}1 & \text { if }\left(\frac{j^{\prime}}{q}\right)=\varepsilon_{1},\left(\frac{\ell^{\prime}}{q}\right)=\varepsilon_{2} \\
0 & \text { otherwise; }\end{cases} \\
\gamma= \begin{cases}1 & \text { if } \nu=\varepsilon \varepsilon_{1}=\varepsilon_{2} \text { or }-\varepsilon=\nu \varepsilon_{1}=1 \\
0 & \text { otherwise. }\end{cases}
\end{gathered}
$$

Proof. By (4) and (15),

$$
\mathcal{T}_{j^{\prime}, \ell^{\prime}}^{\varepsilon_{1}, \varepsilon_{2}}=\mathcal{S}_{-j^{\prime}, \ell^{\prime}}^{\varepsilon \varepsilon_{1}, \varepsilon_{2}}=\lambda \mathcal{S}_{-j, \ell}^{\nu \varepsilon \varepsilon_{1}, \nu \varepsilon_{2}}=\lambda \mathcal{T}_{j, \ell}^{\nu \varepsilon_{1}, \nu \varepsilon_{2}},
$$

so the above sets have the same cardinality $t$, and to prove the result we need only show that $t=m-\beta-\gamma$. Indeed,

$$
\begin{array}{rlrl}
t=\left|\mathcal{S}_{-j, \ell}^{\nu \varepsilon \varepsilon_{1}, \nu \varepsilon_{2}}\right| & =\left|\mathcal{A}_{-j, \ell}^{\nu \varepsilon \varepsilon_{1}, \nu \varepsilon_{2}}\right|-\beta^{\prime} & \text { by Corollary 4.7 } \\
& =m-\gamma^{\prime}-\beta^{\prime} \quad \text { by Proposition 4.6, }
\end{array}
$$

where

$$
\beta^{\prime}=\left\{\begin{array}{ll}
1 & \text { if }\left(\frac{-j}{q}\right)=\nu \varepsilon \varepsilon_{1} \text { and }\left(\frac{\ell}{q}\right)=\nu \varepsilon_{2} \\
0 & \text { otherwise }
\end{array} \quad \gamma^{\prime}= \begin{cases}1 & \text { if } \nu \varepsilon \varepsilon_{1}=\nu \varepsilon_{2}=1 \\
0 & \text { if } \nu \varepsilon \varepsilon_{1}=1=-\nu \varepsilon_{2} \\
(1-\varepsilon) / 2 & \text { if } \nu \varepsilon \varepsilon_{1}=-1 .\end{cases}\right.
$$

First, $\beta=\beta^{\prime}$ because $\left(\frac{-j}{q}\right)=\nu \varepsilon_{1}$ iff $\left(\frac{j^{\prime}}{q}\right)=\varepsilon_{1}$, and $\left(\frac{\ell}{q}\right)=\nu \varepsilon_{2}$ iff $\left(\frac{\ell^{\prime}}{q}\right)=\varepsilon_{2}$. To show that $\gamma=\gamma^{\prime}$, we will show that $\gamma=1$ iff $\gamma^{\prime}=1$ when $\varepsilon=1$, and $\gamma=0$ iff $\gamma^{\prime}=0$ when $\varepsilon=-1$. Suppose $\varepsilon=1$. Then $\gamma^{\prime}=1$ iff $\nu \varepsilon_{1}=\nu \varepsilon_{2}=1$ iff $\nu=\varepsilon_{1}=\varepsilon_{2}$ iff $\gamma=1$. Next, suppose $\varepsilon=-1$. Then $\gamma=1$ iff $\nu=-\varepsilon_{1}=\varepsilon_{2}$ or $\nu=\varepsilon_{1}$. Thus, $\gamma=0$ iff $\nu=-\varepsilon_{1}=-\varepsilon_{2}$ iff $-\nu \varepsilon_{1}=1=-\nu \varepsilon_{2}$ iff $\gamma^{\prime}=0$.

Proposition 5.2 Suppose $j, \ell \in \mathbb{F}_{q}$ and $j+\ell \neq 0$. Then for $\mu \in\{1,-1\}$,

$$
\prod \mathcal{T}_{\ell, j}^{\mu, \mu}= \begin{cases}\mu\left(\frac{2}{q}\right)\left(\frac{j+\ell}{q}\right) \prod \mathcal{T}_{j, \ell}^{\mu, \mu} & \text { if }\left(\frac{j}{q}\right)=\left(\frac{\ell}{q}\right)=\mu \\ -\mu\left(\frac{2}{q}\right)\left(\frac{j+\ell}{q}\right) \prod \mathcal{T}_{j, \ell}^{\mu, \mu} & \text { otherwise. }\end{cases}
$$

Proof. $\mathcal{T}_{\ell, j}^{\mu, \mu}=-\mathcal{T}_{j, \ell}^{\mu, \mu}$ by (書), so $\prod \mathcal{T}_{\ell, j}^{\mu, \mu}=(-1)^{t} \prod \mathcal{T}_{j, \ell}^{\mu, \mu}$, with $t=\left|\mathcal{T}_{j, \ell}^{\mu, \mu}\right|$. By Corollary 4.7, $t=\left|\mathcal{A}_{-j, \ell}^{\varepsilon \mu, \mu}\right|-\beta$, where $\beta=1$ if $\left(\frac{j}{q}\right)=\left(\frac{\ell}{q}\right)=\mu$ and $\beta=0$ otherwise. By (29), $\left|\mathcal{A}_{-j, \ell}^{\varepsilon \mu, \mu}\right|=m-\gamma$, where $\gamma=1$ if $\mu\left(\frac{j+\ell}{q}\right)=1$ and $\gamma=0$ if $\mu\left(\frac{j+\ell}{q}\right)=-1$. Note that $(-1)^{\gamma}=-\mu\left(\frac{j+\ell}{q}\right)$. Thus, by (26) $),(-1)^{t}=(-1)^{m-\gamma-\beta}=\left(\frac{2}{q}\right) \times-\mu\left(\frac{j+\ell}{q}\right)(-1)^{\beta}$. The result follows. 


\section{Legendre symbol identities}

This section presents some results involving Legendre symbols. In an earlier draft of this article, Propositions 6.2 and 6.3 were proved using Theorem 4.1 , but then we found a simpler proof. The results of this section are neither obvious nor deep.

Lemma 6.1 If $a, b, c \in \mathbb{F}_{q}, a b \neq 0$, and $a^{2}+b^{2}=c^{2}$, then $\left(\frac{c+a}{q}\right)=\left(\frac{2}{q}\right)\left(\frac{c+b}{q}\right) \neq 0$. Also, $\left(\frac{c+a}{q}\right)=\left(\frac{c-a}{q}\right)$ and $\left(\frac{c+b}{q}\right)=\left(\frac{c-b}{q}\right)$.

Proof. For the first sentence, it suffices to prove that $2(c+a)(c+b)$ is a nonzero square. It is nonzero because $2(c+a)(c+b)(c-a)(c-b)=2\left(c^{2}-a^{2}\right)\left(c^{2}-b^{2}\right)=2 b^{2} a^{2} \neq 0$. It is a square because

$$
(a+b+c)^{2}=a^{2}+b^{2}+c^{2}+2(a b+a c+b c)=2 c^{2}+2(a b+a c+b c)=2(a+c)(b+c) .
$$

The last sentence follows because $(c+a)(c-a)=b^{2}$ and $(c+b)(c-b)=a^{2}$ are nonzero squares.

Proposition 6.2 If $\tau \in \mathbb{F}_{q}$ and $\left(\frac{\tau(\tau+1)}{q}\right)=1$, then $\left(\frac{2 \tau+1+2 \sqrt{\tau(\tau+1)}}{q}\right)=\left(\frac{\tau}{q}\right)=\left(\frac{\tau+1}{q}\right)$.

Proof. First, $\left(\frac{\tau(\tau+1)}{q}\right)=1$ implies $\left(\frac{\tau}{q}\right)=\left(\frac{\tau+1}{q}\right)$. For the remaining equalities, apply Lemma 6.1 with $a=2 \sqrt{\tau(\tau+1)}, b=1$, and $c=2 \tau+1$.

Proposition 6.3 Suppose that $\tau \in \mathbb{F}_{q}$ and that $\tau, 1+\tau$ are both nonzero squares. Then

(i) $1 / \tau$ and $1+1 / \tau$ are both nonzero squares.

(ii) $1 \pm 1 / \sqrt{1+\tau}$ are both squares or both nonsquares, so the Legendre symbol $\left(\frac{1 \pm 1 / \sqrt{1+\tau}}{q}\right)$ is well defined. Similarly, the Legendre symbol $\left(\frac{1 \pm 1 / \sqrt{1+1 / \tau}}{q}\right)$ is well defined.

(iii) We have

$$
\left(\frac{1 \pm 1 / \sqrt{1+1 / \tau}}{q}\right)=\left(\frac{2}{q}\right)\left(\frac{1 \pm 1 / \sqrt{1+\tau}}{q}\right)
$$

Proof. $(i)$ follows from $1+1 / \tau=(1+\tau) / \tau$. For (ii) and (iii), apply Lemma 6.1 with $a= \pm 1 / \sqrt{1+1 / \tau}, b= \pm 1 / \sqrt{1+\tau}$, and $c=1$. Note that $a^{2}+b^{2}=c^{2}$.

Lemma 6.4 If $j, \ell$ are nonzero squares in $\mathbb{F}_{q}$ and $j+\ell=4$ then

$$
\left(\frac{2 \pm \sqrt{j}}{q}\right)=\left(\frac{2}{q}\right)\left(\frac{2 \pm \sqrt{\ell}}{q}\right) \text {. }
$$

Proof. Apply Lemma 6.1 with $a=\sqrt{j}, b=\sqrt{\ell}$ and $c=2$. 


\section{Formulas for $\prod \mathcal{S}_{k, \ell}^{ \pm, \pm}$and $\prod \mathcal{T}_{j, \ell}^{ \pm, \pm}$}

In evaluating $\prod \mathcal{S}_{k, \ell}^{\varepsilon_{1}, \varepsilon_{2}}$, the rescaling formula (Lemma 5.1) allows to rescale $k$ and $\ell$ by $\lambda \in \mathbb{F}_{q}^{\times}$, and Proposition 3.1 shows how to compute all the values $\prod \mathcal{S}_{k, \ell}^{++}, \prod \mathcal{S}_{k, \ell}^{+-}$, $\prod \mathcal{S}_{k, \ell}^{-+}, \prod \mathcal{S}_{k, \ell}^{--}$if just one of them is known. Thus, we have reduced to computing one example of $\prod \mathcal{S}_{k, \ell}^{ \pm, \pm}$for each ratio $\tau=-k / \ell \in \mathbb{F}_{q} \cup\{\infty\}, \tau \neq-1$. It turns out that we can achieve this, and the technique uses Dickson polynomials. Once the results have been obtained for $\mathcal{S}_{k, \ell}^{ \pm, \pm}$, they can be transferred to results about $\mathcal{T}_{-k, \ell}^{ \pm, \pm}$using (4).

Our policy is to normalize $k$ and $\ell$ for $\mathcal{S}_{k, \ell}^{ \pm, \pm}$so that $\ell-k=4$. That is, given $\tau \neq-1$, we set $k=-4 \tau /(\tau+1)$ and $\ell=4 /(\tau+1)$. We set $r=\ell-2$, so $k=r-2, \ell=r+2$, and $\tau=(2-r) /(2+r)$. For purpose of applying Proposition 3.1, it is useful to note that for $\tau \neq \infty$,

$$
\left(\frac{k}{q}\right)=\varepsilon\left(\frac{\tau}{q}\right)\left(\frac{\tau+1}{q}\right), \quad\left(\frac{\ell}{q}\right)=\left(\frac{\tau+1}{q}\right), \quad\left(\frac{\ell-k}{q}\right)=1 .
$$

It is also convenient to set $\tau^{\prime}=k / 4$. Then $\tau^{\prime}+1=\ell / 4$. When discussing $\mathcal{T}_{j, \ell}^{ \pm, \pm}$, we set $j=-k$, so $\ell+j=4$.

Because these normalizations arise frequently, we highlight the relations between the variables $\tau, k, \ell, r, j, \tau^{\prime}$.

$$
\begin{aligned}
\tau & \in \mathbb{F}_{q} \cup\{\infty\}, \quad \tau \neq-1 \\
k & =-j=-4 \tau /(\tau+1)=4 \tau^{\prime}=r-2 \in \mathbb{F}_{q} \\
\ell & =4+k=4-j=4 /(\tau+1)=4\left(\tau^{\prime}+1\right)=r+2 \in \mathbb{F}_{q} \\
r & =k+2=2-j=\ell-2 \\
\tau^{\prime} & =k / 4=-\tau /(\tau+1), \quad \tau^{\prime}+1=\ell / 4=1 /(\tau+1) \\
\tau & =j / \ell=-k / \ell=(2-r) /(2+r)=-\tau^{\prime} /\left(\tau^{\prime}+1\right)
\end{aligned}
$$

With these normalizations, our formulas for $\prod \mathcal{S}_{k, \ell}^{ \pm, \pm}$and $\prod \mathcal{T}_{j, \ell}^{ \pm, \pm}$are summarized in Theorems 7.1 and 7.2 and Tables 14 below. Theorem 7.1 and Tables 1, 2 handle all cases where $\tau=-k / \ell \in\{0, \infty, 1,1 / 3,3\}$ or where $\tau$ and $\tau+1$ are squares. Theorem 7.2 and Tables 3, 4 handle all cases where $\tau$ and/or $\tau+1$ are nonsquares. In the tables, the conditions on $\tau$ are given on the left, and the corresponding values for normalized $\prod \mathcal{S}_{k, \ell}^{ \pm, \pm}$or $\prod \mathcal{T}_{j, \ell}^{ \pm, \pm}$are given in the columns on the right. In some instances, separate formulas must be given depending on $q \bmod 4, q \bmod 8$, or $q \bmod 12$.

Theorem 7.1 Let $\tau, j, k, \ell$ as in (39)-(37) and $\varepsilon=\left(\frac{-1}{q}\right)$. The following formulas hold for $\prod \mathcal{S}_{k, \ell}^{ \pm, \pm}$and $\prod \mathcal{T}_{j, \ell}^{ \pm, \pm}$when $\tau=j / \ell \in\{0, \infty, 1,1 / 3,3\} \cup \mathcal{A}_{0,1}^{++}$. (For each $\tau$, we provide a formula for one choice of $( \pm, \pm)$, as the remaining ones can be derived from that one using methods of Section 3 . For the reader's convenience, we provide in Tables 1 and 0 the values of $\prod \mathcal{S}_{k, \ell}^{\varepsilon_{1}, \varepsilon_{2}}$ and $\prod \mathcal{T}_{j, \ell}^{\varepsilon_{1}, \varepsilon_{2}}$ for all $\varepsilon_{1}, \varepsilon_{2}$.)

$$
\text { (i) } \tau=0 \text { or } \infty: \quad \prod \mathcal{S}_{0,4}^{-\varepsilon,-}=\prod \mathcal{T}_{0,4}^{--}=\left(\frac{2}{q}\right) \cdot 2 ; \quad \prod \mathcal{S}_{-4,0}^{-\varepsilon,-}=\prod \mathcal{T}_{4,0}^{--}=2 .
$$


(ii) $\tau=1$ : If $q \equiv \pm 1(\bmod 8)$ then $\prod \mathcal{S}_{-2,2}^{-\varepsilon,-}=\prod \mathcal{T}_{2,2}^{--}=(-1)^{(q-\varepsilon) / 8} \cdot 2$, and if $q \equiv \pm 3(\bmod 8)$ then $\prod \mathcal{S}_{-2,2}^{\varepsilon,+}=\prod \mathcal{T}_{2,2}^{++}=(-1)^{(q-\varepsilon-4) / 8}=(-1)^{\lfloor q / 8\rfloor}$.

(iii) $\tau=1 / 3$ or $3: \quad \prod \mathcal{S}_{-1,3}^{-\varepsilon,-}=\prod \mathcal{T}_{1,3}^{--}= \begin{cases}2 & \text { if } q \equiv \pm 1(\bmod 12) \\ -1 & \text { otherwise }\end{cases}$ and $\prod \mathcal{S}_{-3,1}^{-\varepsilon,-}=\prod \mathcal{T}_{3,1}^{--}=\left(\frac{2}{q}\right) \cdot \prod \mathcal{T}_{1,3}^{--}$.

(iv) Suppose that $\tau \in \mathcal{A}_{0,1}^{++}$. (Equivalently, $j$ and $\ell$ are nonzero squares.) Then

$$
\prod \mathcal{S}_{-j, \ell}^{-\varepsilon,-}=\prod \mathcal{T}_{j, \ell}^{--}=\left(\frac{4 \pm 2 \sqrt{\ell}}{q}\right) \cdot 2=\left(\frac{2 \pm \sqrt{j}}{q}\right) \cdot 2 .
$$

(The Legendre symbols in the above formula are well defined and equal to one another by (30).) Also, $\prod \mathcal{T}_{\ell, j}^{--}=\left(\frac{2}{q}\right) \prod \mathcal{T}_{j, \ell}^{--}$.

Theorem 7.1 addresses all cases where $\tau$ and $\tau+1$ are squares. If $\tau$ and/or $\tau+1$ is a nonsquare, then exactly one of $\tau, \tau+1$, and $\tau /(\tau+1)$ is a square. Accordingly, the next theorem gives a formula for $\prod \mathcal{S}_{k, \ell}^{ \pm, \pm}$in terms of a square root of $\tau, \tau+1$, or $\tau /(\tau+1)$. This appears to give the formula only up to a choice of sign, but in Section 8.4 a prescription is given for which square root to take. For each $\tau$, we give $\prod \mathcal{S}_{k, \ell}^{\varepsilon_{1}, \varepsilon_{2}}$ for normalized $k, \ell$ and for one value of $\varepsilon_{1}$ and $\varepsilon_{2}$. The others can be computed by methods of Section 3. For the reader's convenience, we provide in Tables 3 and 4 the values of $\prod \mathcal{S}_{k, \ell}^{\varepsilon_{1}, \varepsilon_{2}}$ and $\prod \mathcal{T}_{-k, \ell}^{\varepsilon_{1}, \varepsilon_{2}}$ for normalized $k$ and $\ell$ and for all $\varepsilon_{1}, \varepsilon_{2}$.

Theorem 7.2 Let $\tau \in \mathbb{F}_{q}, \tau \neq-1, j=-k=4 \tau /(\tau+1), \ell=4 /(\tau+1)$.

(i) Suppose $\tau$ is a nonzero square and $\tau+1$ is a nonsquare. (Equivalently, $j$ and $\ell$ are nonsquares.) Then

$$
\prod \mathcal{T}_{j, \ell}^{++}=\prod \mathcal{S}_{k, \ell}^{\varepsilon,+}=-\left(\frac{2}{q}\right) \cdot \frac{\tau+1}{2 \sqrt{\tau}}=-\left(\frac{2}{q}\right) \cdot \frac{2}{\ell \sqrt{j / \ell}} .
$$

(ii) Suppose $\tau$ is a nonsquare and $\tau+1$ is a square. (Equivalently, $j$ is a nonsquare and $\ell$ is a square.) Then

$$
\prod \mathcal{T}_{j, \ell}^{--}=\prod \mathcal{S}_{k, \ell}^{-\varepsilon,-}=\left(\frac{2}{q}\right) \cdot 2 / \sqrt{\tau+1}=\left(\frac{2}{q}\right) \sqrt{\ell} .
$$

(iii) Suppose $\tau$ and $\tau+1$ are nonsquares. (Equivalently, $j$ is a square and $\ell$ is a nonsquare.) Then

$$
\prod \mathcal{T}_{j, \ell}^{--}=\prod \mathcal{S}_{k, \ell}^{-\varepsilon,-}=2 \sqrt{\tau /(\tau+1)}=\sqrt{j}
$$

In these formulas, the square roots are determined by the prescription given in Section 8.4 . 


\begin{tabular}{|c|c|c|c|c|c|c|c|}
\hline $\begin{array}{c}\text { Condition } \\
\text { on } \tau\end{array}$ & $k$ & $\ell$ & $\begin{array}{l}\text { Condition } \\
\text { on } q\end{array}$ & $\prod \mathcal{S}_{k, \ell}^{++}$ & $\prod \mathcal{S}_{k, \ell}^{+-}$ & $\prod \mathcal{S}_{k, \ell}^{-+}$ & $\prod \mathcal{S}_{k, \ell}^{--}$ \\
\hline \multirow{2}{*}{$\tau=0$} & \multirow{2}{*}{0} & \multirow{2}{*}{4} & $1(\bmod 4)$ & $\left(\frac{2}{q}\right) / 4$ & $\left(\frac{2}{q}\right)$ & $\left(\frac{2}{q}\right) / 2$ & $\left(\frac{2}{q}\right) \cdot 2$ \\
\hline & & & $3(\bmod 4)$ & $\left(\frac{2}{q}\right) / 2$ & $\left(\frac{2}{q}\right) \cdot 2$ & $-\left(\frac{2}{q}\right) / 4$ & $-\left(\frac{2}{q}\right)$ \\
\hline \multirow{2}{*}{$\tau=\infty$} & \multirow{2}{*}{-4} & \multirow{2}{*}{0} & $1(\bmod 4)$ & $-1 / 4$ & $1 / 2$ & 1 & 2 \\
\hline & & & $3(\bmod 4)$ & 1 & 2 & $1 / 4$ & $-1 / 2$ \\
\hline \multirow{4}{*}{$\tau=1$} & \multirow{4}{*}{-2} & \multirow{4}{*}{2} & $1(\bmod 8)$ & $(-1)^{(q-1) / 8} / 8$ & $(-1)^{(q-1) / 8}$ & $(-1)^{(q-1) / 8}$ & $(-1)^{(q-1) / 8} \cdot 2$ \\
\hline & & & $5(\bmod 8)$ & $(-1)^{(q-5) / 8}$ & $(-1)^{(q-5) / 8}$ & $(-1)^{(q+3) / 8}$ & $(-1)^{(q+3) / 8} / 4$ \\
\hline & & & $3(\bmod 8)$ & $(-1)^{(q-3) / 8}$ & $(-1)^{(q-3) / 8} / 4$ & $(-1)^{(q-3) / 8}$ & $(-1)^{(q-3) / 8}$ \\
\hline & & & $7(\bmod 8)$ & $(-1)^{(q+1) / 8}$ & $(-1)^{(q+1) / 8} \cdot 2$ & $(-1)^{(q-7) / 8} / 8$ & $(-1)^{(q-7) / 8}$ \\
\hline \multirow{4}{*}{$\tau=3$} & \multirow{4}{*}{-3} & \multirow{4}{*}{1} & $1(\bmod 12)$ & $\left(\frac{2}{q}\right) / 6$ & $\left(\frac{2}{q}\right)$ & $\left(\frac{2}{q}\right)$ & $\left(\frac{2}{q}\right) \cdot 2$ \\
\hline & & & $5(\bmod 12)$ & $-\left(\frac{2}{q}\right)$ & $-\left(\frac{2}{q}\right) \cdot 2$ & $-\left(\frac{2}{q}\right) / 6$ & $-\left(\frac{2}{q}\right)$ \\
\hline & & & $7(\bmod 12)$ & $-\left(\frac{2}{q}\right) / 6$ & $-\left(\frac{2}{q}\right)$ & $\left(\frac{2}{q}\right)$ & $\left(\frac{2}{q}\right) \cdot 2$ \\
\hline & & & $11(\bmod 12)$ & $\left(\frac{2}{q}\right)$ & $\left(\frac{2}{q}\right) \cdot 2$ & $-\left(\frac{2}{q}\right) / 6$ & $-\left(\frac{2}{q}\right)$ \\
\hline \multirow{4}{*}{$\tau=1 / 3$} & \multirow{4}{*}{-1} & \multirow{4}{*}{3} & $1(\bmod 12)$ & $1 / 6$ & 1 & 1 & 2 \\
\hline & & & $5(\bmod 12)$ & 1 & $1 / 6$ & -2 & -1 \\
\hline & & & $7(\bmod 12)$ & -2 & -1 & -1 & $-1 / 6$ \\
\hline & & & $11(\bmod 12)$ & 1 & 2 & $-1 / 6$ & -1 \\
\hline \multirow{2}{*}{$\begin{array}{c}\tau, \tau+1, \text { and } \\
1 \pm 1 / \sqrt{\tau+1} \\
\text { are squares }\end{array}$} & \multirow[b]{2}{*}{$\frac{-4 \tau}{\tau+1}$} & \multirow[b]{2}{*}{$\frac{4}{\tau+1}$} & $1(\bmod 4)$ & $-1 /(2 k \ell)$ & 1 & 1 & 2 \\
\hline & & & $3(\bmod 4)$ & 1 & 2 & $1 /(2 k \ell)$ & -1 \\
\hline $\begin{array}{c}\tau, \tau+1 \text { are } \\
\text { squares; }\end{array}$ & \multirow{2}{*}{$\frac{-4 \tau}{\tau+1}$} & \multirow{2}{*}{$\frac{4}{\tau+1}$} & $1(\bmod 4)$ & $1 /(2 k \ell)$ & -1 & -1 & -2 \\
\hline $\begin{array}{l}1 \pm 1 / \sqrt{\tau+1} \\
\text { are nonsquares }\end{array}$ & & & $3(\bmod 4)$ & -1 & -2 & $(-1) /(2 k \ell)$ & 1 \\
\hline
\end{tabular}

Table 1: $\prod \mathcal{S}_{k, \ell}^{ \pm, \pm}$for values of $\tau=-k / \ell$ given in Theorem 7.1 . Here $k$ and $\ell$ are normalized so that $\ell-k=4$. One entry from each row is proved in Theorem 7.1, and the remaining entries of the row can be deduced using methods of Section 3 . In the bottom two rows, we note that $1 \pm 1 / \sqrt{\tau+1}$ are squares iff $2 \pm 2 \sqrt{\tau /(\tau+1)}$ are squares by Proposition 6.3. Also, $1 \pm 1 / \sqrt{\tau+1}=1 \pm \sqrt{\ell} / 2$ and $2 \pm 2 \sqrt{\tau /(\tau+1)}=$ $2 \pm \sqrt{-k}$. 


\begin{tabular}{|c|c|c|c|c|c|c|}
\hline $\begin{array}{c}\text { Condition } \\
\text { on } \tau \text { and } q\end{array}$ & $j$ & $\ell$ & $\prod \mathcal{T}_{j, \ell}^{++}$ & $\prod \mathcal{T}_{j, \ell}^{+-}$ & $\prod \mathcal{T}_{j, \ell}^{-+}$ & $\prod \mathcal{T}_{j, \ell}^{--}$ \\
\hline \hline$\tau=0$ & 0 & 4 & $\left(\frac{-2}{q}\right) / 4$ & $\left(\frac{-2}{q}\right)$ & $\left(\frac{2}{q}\right) / 2$ & $\left(\frac{2}{q}\right) \cdot 2$ \\
\hline$\tau=\infty$ & 4 & 0 & $-\left(\frac{-1}{q}\right) / 4$ & $\left(\frac{-1}{q}\right) / 2$ & 1 & 2 \\
\hline $\begin{array}{c}\tau=1 \\
q \equiv \pm 1(\bmod 8)\end{array}$ & 2 & 2 & $(-1)^{\lfloor q / 8\rfloor} / 8$ & $(-1)^{\lfloor q / 8\rfloor}$ & $(-1)^{\lfloor(q+3) / 8\rfloor}$ & $(-1)^{\lfloor(q+3) / 8\rfloor} \cdot 2$ \\
\hline $\begin{array}{c}\tau=1 \\
q= \pm 3(\bmod 8)\end{array}$ & 2 & 2 & $(-1)^{\lfloor q / 8\rfloor}$ & $(-1)^{\lfloor q / 8\rfloor}$ & $(-1)^{\lfloor(q+3) / 8\rfloor}$ & $(-1)^{\lfloor(q+3) / 8\rfloor} / 4$ \\
\hline $\begin{array}{c}\tau=3 \\
q \equiv \pm 1(\bmod 12)\end{array}$ & 3 & 1 & $\left(\frac{-2}{q}\right) / 6$ & $\left(\frac{-2}{q}\right)$ & $\left(\frac{2}{q}\right)$ & $\left(\frac{2}{q}\right) \cdot 2$ \\
\hline $\begin{array}{c}\tau=3 \\
q \equiv \pm 5(\bmod 12)\end{array}$ & 3 & 1 & $-\left(\frac{-2}{q}\right)$ & $-\left(\frac{-2}{q}\right) \cdot 2$ & $-\left(\frac{2}{q}\right) / 6$ & $-\left(\frac{2}{q}\right)$ \\
\hline $\begin{array}{c}\tau=1 / 3 \\
q \equiv \pm 1(\bmod 12)\end{array}$ & 1 & 3 & $\left(\frac{-1}{q}\right) / 6$ & $\left(\frac{-1}{q}\right)$ & 1 & 2 \\
\hline $\begin{array}{c}\tau=1 / 3 \\
q \equiv \pm 5(\bmod 12)\end{array}$ & 1 & 3 & $\left(\frac{-1}{q}\right)$ & $\left(\frac{-1}{q}\right) / 6$ & -2 & -1 \\
\hline $\begin{array}{c}\tau, \tau+1, \text { and } \\
1 \pm 1 / \sqrt{\tau+1} \\
\text { are nonzero squares }\end{array}$ & $\frac{4 \tau}{\tau+1}$ & $\frac{4}{\tau+1}$ & $\left(\frac{-1}{q}\right) /(2 j \ell)$ & $\left(\frac{-1}{q}\right)$ & 1 & -2 \\
\hline $\begin{array}{c}\tau, 1 \text { are } \\
1 \pm 1 / \sqrt{\tau+1} \\
\text { are nonsquares }\end{array}$ & $\frac{4 \tau}{\tau+1}$ & $\frac{4}{\tau+1}$ & $-\left(\frac{-1}{q}\right) /(2 j \ell)$ & $-\left(\frac{-1}{q}\right)$ & -1 & 2 \\
\hline
\end{tabular}

Table 2: $\quad \prod \mathcal{T}_{j, \ell}^{ \pm, \pm}$for values of $\tau=j / \ell$ given in Theorem 7.1. Here $j$ and $\ell$ are normalized so that $j+\ell=4$, thus $j=4 \tau /(\tau+1)$ and $\ell=4 /(\tau+1)$. In the bottom two rows of the table, note that $1 \pm 1 / \sqrt{\tau+1}$ is a square iff $2 \pm 2 \sqrt{\tau /(\tau+1)}$ is a square by Proposition 6.3. Also note that $1 \pm 1 / \sqrt{\tau+1}=1 \pm \sqrt{\ell} / 2$ and $2 \pm 2 \sqrt{\tau /(\tau+1)}=2 \pm \sqrt{j}$.

Formula (5) of the introduction can be found in the row " $\tau=1$ " of Table 2. Formula (6) follows from the row " $\tau=1 / 3$ " of Table 2 if $q$ is not a power of 3 , and from the row " $\tau=\infty "$ of Table 2 if $q$ is a power of 3. Formulas (9) and (8) follow from Theorem 7.2 ( $i i)$ and (iii). Formula (10) follows from Theorem $7.2(i)$ and Proposition 3.1 (or see Table 4). 


\begin{tabular}{|c|c|c|c|c|c|c|}
\hline $\begin{array}{c}\text { Condition } \\
\text { on } \tau\end{array}$ & Value of $c$ & $\begin{array}{c}\text { Condition } \\
\text { on } q\end{array}$ & $\prod \mathcal{S}_{k, \ell}^{++}$ & $\prod \mathcal{S}_{k, \ell}^{+-}$ & $\prod \mathcal{S}_{k, \ell}^{-+}$ & $\prod \mathcal{S}_{k, \ell}^{--}$ \\
\hline \hline $\begin{array}{c}\left(\frac{\tau}{q}\right)=1, \\
\left(\frac{\tau+1}{q}\right)=-1\end{array}$ & \multirow{2}{*}{$\left(\frac{2}{q}\right) \sqrt{\tau}$} & $1(\bmod 4)$ & $\frac{-(\tau+1)}{2 c}$ & $-c$ & $1 / c$ & $\frac{\tau+1}{8 c}$ \\
\cline { 3 - 7 } & $3(\bmod 4)$ & $-1 / c$ & $\frac{-(\tau+1)}{8 c}$ & $\frac{-(\tau+1)}{2 c}$ & $-c$ \\
\hline $\begin{array}{c}\left(\frac{\tau}{q}\right)=-1, \\
\left(\frac{\tau+1}{q}\right)=1\end{array}$ & \multirow{2}{*}{$\left(\frac{2}{q}\right) \sqrt{\tau+1}$} & $1(\bmod 4)$ & $c / 2$ & $c$ & $\frac{c(\tau+1)}{16 \tau}$ & $2 / c$ \\
\cline { 3 - 7 } & $3(\bmod 4)$ & $\frac{c(\tau+1)}{16 \tau}$ & $2 / c$ & $-c / 2$ & $-c$ \\
\hline$\left(\frac{\tau}{q}\right)=-1$, & $\sqrt{\frac{\tau}{\tau+1}}$ & $1(\bmod 4)$ & $-1 /(2 c)$ & $\frac{-(\tau+1)}{16 c}$ & $1 / c$ & $2 c$ \\
\cline { 3 - 7 }$\left(\frac{\tau+1}{q}\right)=-1$ & $3(\bmod 4)$ & $1 / c$ & $2 c$ & $1 /(2 c)$ & $\frac{\tau+1}{16 c}$ \\
\hline
\end{tabular}

Table 3: $\prod \mathcal{S}_{k, \ell}^{ \pm, \pm}$for values of $\tau=-k / \ell$ given in Theorem 7.2. Here $k=-4 \tau /(\tau+1)$ and $\ell=4 /(\tau+1)$. In each case, $\tau \in \mathbb{F}_{q} \backslash\{0,-1\}$, and exactly one of $\tau, \tau+1$, or $\tau /(\tau+1)$ is a square. Accordingly, we let $c \in \mathbb{F}_{q}$ denote a square root of $\tau, \tau+1$, or $\tau /(\tau+1)$. The choice for $\sqrt{\tau}, \sqrt{\tau+1}$, or $\sqrt{\tau /(\tau+1)}$ is prescribed in Section 8.4.

\begin{tabular}{|c|c|c|c|c|c|}
\hline Condition on $\tau$ & $c$ & $\prod \mathcal{T}_{j, \ell}^{++}$ & $\prod \mathcal{T}_{j, \ell}^{+-}$ & $\prod \mathcal{T}_{j, \ell}^{-+}$ & $\prod \mathcal{T}_{j, \ell}^{--}$ \\
\hline \hline$\left(\frac{\tau}{q}\right)=1,\left(\frac{\tau+1}{q}\right)=-1$ & $\left(\frac{2}{q}\right) \sqrt{\tau}$ & $\frac{-(\tau+1)}{2 c}$ & $-c$ & $\varepsilon / c$ & $\frac{\varepsilon(\tau+1)}{8 c}$ \\
\hline$\left(\frac{\tau}{q}\right)=-1,\left(\frac{\tau+1}{q}\right)=1$ & $\left(\frac{2}{q}\right) \sqrt{\tau+1}$ & $\varepsilon c / 2$ & $\varepsilon c$ & $\frac{c(\tau+1)}{16 \tau}$ & $2 / c$ \\
\hline$\left(\frac{\tau}{q}\right)=\left(\frac{\tau+1}{q}\right)=-1$ & $\sqrt{\frac{\tau}{\tau+1}}$ & $-\varepsilon /(2 c)$ & $\frac{\varepsilon(\tau+1)}{-16 c}$ & $1 / c$ & $2 c$ \\
\hline
\end{tabular}

Table 4: $\prod \mathcal{T}_{j, \ell}^{ \pm, \pm}$for values of $\tau=j / \ell$ given in Theorem 7.2. Here $j=4 \tau /(\tau+1)$, $\ell=4 /(\tau+1)$, and $\varepsilon=\left(\frac{-1}{q}\right)=(-1)^{(q-1) / 2}$. In each case, $\tau \in \mathbb{F}_{q} \backslash\{0,-1\}$ and exactly one of $\tau, \tau+1$, or $\tau /(\tau+1)$ is a square. Accordingly, we let $c \in \mathbb{F}_{q}$ denote a square root of $\tau, \tau+1$, or $\tau /(\tau+1)$. The choice for $\sqrt{\tau}, \sqrt{\tau+1}$, or $\sqrt{\tau /(\tau+1)}$ is prescribed in Section 8.4. 


\section{Proofs of Theorems 7.1 and $\mathbf{7 . 2}$}

Our main tool to prove formulas for $\prod \mathcal{S}_{k, \ell}^{ \pm, \pm}$is a result from [1] about Dickson polynomials. Section 8.1 provides a brief background on Dickson polynomials and recalls relevant results from [1]. Section 8.2 uses those results to prove Theorem 7.1( $(i)-$ (iii). Section 8.3 relates $\tau$ with two other parameters $r$ and $u$. Section 8.4 describes a way to uniquely determine square roots of $\tau, \tau+1$, or $\tau /(\tau+1)$ in the case where exactly one of these three is a square. Then in Section 8.5 we prove Theorem 7.1 $(\mathrm{iv})$ and Theorem 7.2

\subsection{Using Dickson polynomials to compute $\prod \mathcal{S}_{k, \ell}^{ \pm, \pm}$}

Dickson polynomials, introduced in Leonard Eugene Dickson's PhD thesis in 1896, are closely related to Chebyshev polynomials and have been widely studied; see [5]. Dickson polynomials of the first kind, $D_{k}(x) \in \mathbb{Z}[x]$, are determined from the recursion

$$
D_{0}(x)=2, D_{1}(x)=x \text {, and } D_{k+2}(x)=x D_{k+1}(x)-D_{k}(x) .
$$

Dickson polynomials of the second kind, $E_{k}(x) \in \mathbb{Z}[x]$, are determined from the recursion

$$
E_{0}(x)=1, E_{1}(x)=x, \text { and } E_{k+2}(x)=x E_{k+1}(x)-E_{k}(x) .
$$

It is well known, and can easily be shown by induction, that $D_{k}$ and $E_{k}$ satisfy the functional equations

$$
D_{k}(\langle u\rangle)=\left\langle u^{k}\right\rangle, \quad E_{k-1}(\langle u\rangle)=\frac{u^{k}-1 / u^{k}}{u-1 / u}
$$

where we use the notation

$$
\langle u\rangle=u+1 / u .
$$

Define functions $f^{\varepsilon_{1}, \varepsilon_{2}}(x) \in \mathbb{F}_{q}[x]$ by

$$
f^{\varepsilon_{1}, \varepsilon_{2}}(x)=\prod\left\{(x-b): b \in \mathcal{A}_{-2,2}^{\varepsilon_{1}, \varepsilon_{2}}\right\} .
$$

Theorem 8.1 ([1] Let $\varepsilon=\left(\frac{-1}{q}\right)$, so $\varepsilon \in\{+1,-1\}$ and $q \equiv \varepsilon(\bmod 4)$. In $\mathbb{F}_{q}[x]$ we have

$$
D_{(q-\varepsilon) / 4}(x)=f^{-\varepsilon,-}(x), \quad E_{(q-\varepsilon-4) / 4}(x)=f^{\varepsilon,+}(x) .
$$

Proof. Although this was proved in [1], we give here a slightly different proof that is based on Corollary 4.2, Let $m=(q-\varepsilon) / 4$.

(i) $\left|\mathcal{A}_{-2,2}^{-\varepsilon,-}\right|=m$ by (29), so $D_{m}(x)$ and $f^{-\varepsilon,-}(x)$ are monic polynomials of the same degree. Thus, it suffices to prove that $D_{m}$ vanishes at all $b \in \mathcal{A}_{-2,2}^{-\varepsilon,-}$. By Corollary 4.2, $b=\left\langle v^{2}\right\rangle$ where $v^{q-\varepsilon}=-1$ and $v^{4} \neq 1$. Then $i=v^{(q-\varepsilon) / 2}$ is a square root of -1 , and

$$
D_{m}(b)=D_{m}\left(\left\langle v^{2}\right\rangle\right)=\left\langle v^{2 m}\right\rangle=\left\langle v^{(q-\varepsilon) / 2}\right\rangle=\langle i\rangle=0 .
$$

(ii) By (29),$\left|\mathcal{A}_{-2,2}^{\varepsilon,+}\right|=m-1$, so $E_{m-1}(x)$ and $f^{\varepsilon,+}(x)$ are monic polynomials of the same degree. To show $E_{m-1}=f^{\varepsilon,+}$, it suffices to show $E_{m-1}(b)=0$ for all $b \in \mathcal{A}_{-2,2}^{\varepsilon,+}$. By Corollary 4.2, $b=\left\langle v^{2}\right\rangle$, where $v^{q-\varepsilon}=1$ and $v^{4} \neq 1$. Then

$$
E_{m-1}(b)=E_{m-1}\left(\left\langle v^{2}\right\rangle\right)=\left(\left(v^{2}\right)^{m}-\left(v^{2}\right)^{-m}\right) /\left(v^{2}-v^{-2}\right) .
$$


Here the denominator does not vanish, since $v^{4} \neq 1$. On the other hand $v^{2 m} \in\{1,-1\}$ since its square is 1 , so the numerator does vanish, and $E_{m-1}(b)=0$.

Example 1. Consider $q=13$. The nonzero squares in $\mathbb{F}_{13}$ are $1,3,4,9,10,12$ and the nonsquares are $2,5,6,7,8,11$. Thus, $\mathcal{A}_{-2,2}^{++}=\{1,12\}, \mathcal{A}_{-2,2}^{+-}=\{3,5,6\}, \mathcal{A}_{-2,2}^{-+}=$ $\{7,8,10\}, \mathcal{A}_{-2,2}^{--}=\{4,9,0\}$. We have $f^{++}(x)=(x-1)(x-12) \equiv x^{2}-1=E_{2}(x)$ $(\bmod 13)$ and $f^{--}(x)=(x-4)(x-9)(x-0) \equiv x^{3}-3 x=D_{3}(x)(\bmod 13)$.

Example 2. Consider $q=23$. The nonzero squares in $\mathbb{F}_{23}$ are $1,2,3,4,6,8,9,12,13,16,18$ and the nonsquares are $5,7,10,11,14,15,17,19,20,21,22$. Thus,

$$
\begin{aligned}
\mathcal{A}_{-2,2}^{++} & =\{4,6,10,11,14\}, & \mathcal{A}_{-2,2}^{+-} & =\{3,5,8,15,18,20\}, \\
\mathcal{A}_{-2,2}^{-+} & =\{0,1,7,16,22\}, & \mathcal{A}_{-2,2}^{--} & =\{9,12,13,17,19\} .
\end{aligned}
$$

Then $f^{-+}(x)=x(x-1)(x-7)(x-16)(x-22) \equiv x^{5}-4 x^{3}+3 x=E_{5}(x)(\bmod 23)$,

$f^{+-}(x)=(x-3)(x-5)(x-8)(x-15)(x-18)(x-20) \equiv x^{6}-6 x^{4}+9 x^{2}-2=D_{6}(x) \quad(\bmod 23)$.

Lemma 8.2 If $r \in \mathbb{F}_{q} \backslash \mathcal{A}_{-2,2}^{\varepsilon_{1}, \varepsilon_{2}}$ then $(-1)^{\left|\mathcal{A}_{-2,2}^{\varepsilon_{1}, \varepsilon_{2}}\right|} f^{\varepsilon_{1}, \varepsilon_{2}}(r)=\prod \mathcal{S}_{r-2, r+2}^{\varepsilon_{1}, \varepsilon_{2}}$.

Proof. The set of roots of $f^{\varepsilon_{1}, \varepsilon_{2}}$ is precisely $\mathcal{A}_{-2,2}^{\varepsilon_{1}, \varepsilon_{2}}$, so the hypothesis that $r \notin \mathcal{A}_{-2,2}^{\varepsilon_{1}, \varepsilon_{2}}$ implies that $f^{\varepsilon_{1}, \varepsilon_{2}}(r) \neq 0$. We have

$$
\begin{aligned}
& f^{\varepsilon_{1}, \varepsilon_{2}}(r)=\prod\left\{r-b: b \in \mathcal{A}_{-2,2}^{\varepsilon_{1}, \varepsilon_{2}}\right\}, \\
(-1)^{\left|\mathcal{A}_{-2,2}^{\varepsilon_{1}, \varepsilon_{2}}\right|} f^{\varepsilon_{1}, \varepsilon_{2}}(r)= & \prod\left\{b-r: b \in \mathcal{A}_{-2,2}^{\varepsilon_{1}, \varepsilon_{2}}\right\} \\
= & \prod\left\{a: a+r \in \mathcal{A}_{-2,2}^{\varepsilon_{1}, \varepsilon_{2}}\right\} \\
= & \prod\left\{a \in \mathbb{F}_{q}:\left(\frac{a+r-2}{q}\right)=\varepsilon_{1}, \quad\left(\frac{a+r+2}{q}\right)=\varepsilon_{2}\right\} .
\end{aligned}
$$

Since this is nonzero, 0 does not occur in the product, and so this equals

$$
\prod\left\{a \in \mathbb{F}_{q}^{\times}:\left(\frac{a+r-2}{q}\right)=\varepsilon_{1},\left(\frac{a+r+2}{q}\right)=\varepsilon_{2}\right\}=\prod \mathcal{S}_{r-2, r+2}^{\varepsilon_{1}, \varepsilon_{2}}
$$

as claimed.

Proposition 8.3 Let $r \in \mathbb{F}_{q}$ and write $r=\langle u\rangle$, where $u \in \overline{\mathbb{F}}_{q}^{\times}$. Let $\varepsilon=\left(\frac{-1}{q}\right)$.

(i) If $r \notin \mathcal{A}_{-2,2}^{-\varepsilon,-}$, then $\prod \mathcal{S}_{r-2, r+2}^{-\varepsilon,-}=\left\langle(-u)^{(q-\varepsilon) / 4}\right\rangle=\left(\frac{2}{q}\right)\left\langle u^{(q-\varepsilon) / 4}\right\rangle$.

(ii) If $r \notin \mathcal{A}_{-2,2}^{\varepsilon,+} \cup\{2,-2\}$, then

$$
\begin{aligned}
\prod \mathcal{S}_{r-2, r+2}^{\varepsilon,+} & =-\left((-u)^{(q-\varepsilon) / 4}-(-u)^{-(q-\varepsilon) / 4}\right) /(u-1 / u) \\
& =-\left(\frac{2}{q}\right)\left(u^{(q-\varepsilon) / 4}-u^{-(q-\varepsilon) / 4}\right) /(u-1 / u) .
\end{aligned}
$$


Proof. (i) Suppose $r \in \mathbb{F}_{q} \backslash \mathcal{A}_{-2,2}^{-\varepsilon,-}$. Then

$$
\begin{aligned}
\prod \mathcal{S}_{r-2, r+2}^{-\varepsilon,-} & =(-1)^{\left|\mathcal{A}_{-2,2}^{-\varepsilon,-}\right|} f^{-\varepsilon,-}(r) \quad \text { by Lemma 8.2 } \\
& =(-1)^{(q-\varepsilon) / 4} D_{(q-\varepsilon) / 4}(r) \quad \text { by Theorem 8.1 and (29) } \\
& =(-1)^{(q-\varepsilon) / 4}\left\langle u^{(q-\varepsilon) / 4}\right\rangle \quad \text { by }(\underline{40}) \\
& =\left\langle(-u)^{(q-\varepsilon) / 4}\right\rangle \quad \text { since }-\langle v\rangle=\langle-v\rangle=-(v+1 / v) .
\end{aligned}
$$

By (26), we also have $\prod \mathcal{S}_{r-2, r+2}^{-\varepsilon,-}=\left(\frac{2}{q}\right)\left\langle u^{(q-\varepsilon) / 4}\right\rangle$.

(ii) Suppose $r \notin \mathcal{A}_{-2,2}^{\varepsilon,+} \cup\{2,-2\}$. Note that $r \notin\{2,-2\}$ implies $u \notin\{1,-1\}$, so in particular $u-1 / u \neq 0$. We have

$$
\begin{aligned}
\prod \mathcal{S}_{r-2, r+2}^{\varepsilon,+} & =(-1)^{\left|\mathcal{A}_{-2,2}^{\varepsilon,+}\right|} f^{\varepsilon,+}(r) \quad \text { by Lemma } 8.2 \\
& =(-1)^{(q-\varepsilon-4) / 4} E_{(q-\varepsilon-4) / 4}(r) \quad \text { by Theorem } 8.1 \text { and (29) } \\
& =(-1)^{(q-\varepsilon-4) / 4}\left(u^{(q-\varepsilon) / 4}-u^{-(q-\varepsilon) / 4}\right) /(u-1 / u) \quad \text { by (40) } \\
& =-\left((-u)^{(q-\varepsilon) / 4}-(-u)^{-(q-\varepsilon) / 4}\right) /(u-1 / u) .
\end{aligned}
$$

The right side is also equal to $-\left(\frac{2}{q}\right)\left(u^{(q-\varepsilon) / 4}-u^{-(q-\varepsilon) / 4}\right) /(u-1 / u)$ by (26) $)$.

\subsection{Proof of Theorem $\mathbf{7 . 1}(i)-(i i i)$.}

We are ready to prove parts $(i)-($ iii $)$ of Theorem 7.1 ,

(i) $\tau=0$ : Set $r=2=\langle 1\rangle$. By Proposition $\left[8.3(i), \prod \mathcal{S}_{0,4}^{-\varepsilon,-}=\left(\frac{2}{q}\right)\langle 1\rangle=\left(\frac{2}{q}\right) \cdot 2\right.$.

$\tau=\infty$ : Set $r=-2=\langle-1\rangle$. By Proposition $8.3(i), \prod \mathcal{S}_{-4,0}^{-\varepsilon,-}=\langle 1\rangle=2$.

(ii) $\tau=1$ : Set $r=0=\langle i\rangle$, where $i^{2}=-1$.

If $q \equiv \pm 1(\bmod 8)$ then $\left(\frac{2}{q}\right)=1$ by Lemma 4.3, so $0 \notin \mathcal{A}_{-2,2}^{-\varepsilon,-}$. By Proposition $8.3(i)$,

$$
\prod \mathcal{S}_{-2,2}^{-\varepsilon,-}=\left\langle(-i)^{(q-\varepsilon) / 4}\right\rangle=\left\langle(-1)^{(q-\varepsilon) / 8}\right\rangle=(-1)^{(q-\varepsilon) / 8} \cdot 2
$$

If $q \equiv \pm 3(\bmod 8)$ then $\left(\frac{2}{q}\right)=-1$, so $0 \notin \mathcal{A}_{-2,2}^{\varepsilon,+}$. By Proposition 8.3) $(i i)$,

$$
\prod \mathcal{S}_{-2,2}^{\varepsilon,+}=\left(i^{(q-\varepsilon) / 4}-i^{-(q-\varepsilon) / 4}\right) /(i-1 / i)
$$

Write $q=8 m+4+\varepsilon$. Since $i^{(q-\varepsilon) / 4}=(-1)^{m} \cdot i$, the right side is $(-1)^{m}(i-1 / i) /(i-1 / i)=$ $(-1)^{m}$. Thus, $\prod \mathcal{S}_{-2,2}^{\varepsilon,+}=(-1)^{(q-4-\varepsilon) / 8}=(-1)^{\lfloor q / 8\rfloor}$.

(iii) $\tau=1 / 3$ : Set $r=1=\langle-\omega\rangle$, where $\omega$ is a primitive cube root of unity. Then $\left(\frac{r-2}{q}\right)=\left(\frac{-1}{q}\right)=\varepsilon$, so $r \notin \mathcal{A}_{-2,2}^{-\varepsilon,-}$. By Proposition [8.3, $\prod \mathcal{S}_{-1,3}^{-\varepsilon,-}=\left\langle\omega^{m}\right\rangle$, where $m=(q-\varepsilon) / 4$. This equals 2 if $3 \mid m$, or -1 if $3 \nmid m$. Here $3 \mid m \Longleftrightarrow q \equiv \pm 1(\bmod 12)$. $\tau=3: \prod \mathcal{S}_{-3,1}^{-\varepsilon,-}=\prod \mathcal{T}_{3,1}^{--}=\left(\frac{2}{q}\right) \prod \mathcal{T}_{1,3}^{--}$by (4) and Proposition 5.2 .

Remark. If $q \equiv \pm 3(\bmod 12)$ (i.e. $\left.q=3^{n}\right)$, then $\mathcal{S}_{-3,1}^{\varepsilon_{1}, \varepsilon_{2}}=\mathcal{S}_{0,4}^{\varepsilon_{1}, \varepsilon_{2}}$ and $\mathcal{T}_{3,1}^{\varepsilon_{1}, \varepsilon_{2}}=\mathcal{T}_{0,4}^{\varepsilon_{1}, \varepsilon_{2}}$ because $0=3$ and $1=4$ in $\mathbb{F}_{q}$. For this reason, we exclude the cases $q \equiv \pm 3(\bmod 12)$ from Tables 1 and 2 when $\tau=1 / 3$ or $\tau=3$. 


\subsection{Relation between $\tau, r$, and $u$.}

Let $\tau \in \mathbb{F}_{q} \backslash\{0,-1\}, k, \ell, r, \tau^{\prime}$ be as in equations (32)-(37) and $\varepsilon=\left(\frac{-1}{q}\right)$. Note that $\tau^{\prime} \in \mathbb{F}_{q} \backslash\{0,-1\}$.

Lemma 8.4 Let $v=\sqrt{\tau+1}+\sqrt{\tau}$ and $w=\sqrt{\tau^{\prime}}+\sqrt{\tau^{\prime}+1}$ (for any choice of square roots). Then $v^{4} \neq 1, w^{4} \neq 1$, and $r=\left\langle w^{2}\right\rangle$. For $\varepsilon_{1}, \varepsilon_{2} \in\{1,-1\}$ we have the following equivalence:

$\tau \in \mathcal{A}_{0,1}^{\varepsilon_{1}, \varepsilon_{2}} \Longleftrightarrow \tau^{\prime} \in \mathcal{A}_{0,1}^{\varepsilon \varepsilon_{1} \varepsilon_{2}, \varepsilon_{2}} \Longleftrightarrow r \in \mathcal{A}_{-2,2}^{\varepsilon \varepsilon_{1} \varepsilon_{2}, \varepsilon_{2}} \Longleftrightarrow v^{q-\varepsilon_{1} \varepsilon_{2}}=\varepsilon_{2} \Longleftrightarrow w^{q-\varepsilon \varepsilon_{1}}=\varepsilon_{2}$.

Proof. As noted in (23), $\mathbb{F}_{q} \backslash\{0,-1\}$ is a disjoint union of the sets $\mathcal{A}_{0,1}^{\varepsilon_{1}, \varepsilon_{2}}$. By Theorem 4.1, $\tau \in \mathcal{A}_{0,1}^{\varepsilon_{1}, \varepsilon_{2}}$ iff $v^{q-\varepsilon_{1} \varepsilon_{2}}=\varepsilon_{2}$ and $v^{4} \neq 1$. By (36),$\tau \in \mathcal{A}_{0,1}^{\varepsilon_{1}, \varepsilon_{2}}$ iff $\tau^{\prime} \in \mathcal{A}_{0,1}^{\varepsilon \varepsilon_{1} \varepsilon_{2}, \varepsilon_{2}}$. By Theorem 4.1, $\tau^{\prime} \in \mathcal{A}_{0,1}^{\varepsilon \varepsilon_{1} \varepsilon_{2}, \varepsilon_{2}}$ iff $w^{q-\varepsilon \varepsilon_{1}}=\varepsilon_{2}$ and $w^{4} \neq 1$. Since $r=4 \tau^{\prime}+2$, we have $\left(\frac{r-2}{q}\right)=\left(\frac{\tau^{\prime}}{q}\right)$ and $\left(\frac{r+2}{q}\right)=\left(\frac{\tau^{\prime}+1}{q}\right)$, so $\tau^{\prime} \in \mathcal{A}_{0,1}^{\varepsilon \varepsilon_{1} \varepsilon_{2}, \varepsilon_{2}}$ iff $r \in \mathcal{A}_{-2,2}^{\varepsilon \varepsilon_{1} \varepsilon_{2}, \varepsilon_{2}}$. It remains only to show that $r=\left\langle w^{2}\right\rangle$. Indeed,

$$
\left\langle w^{2}\right\rangle=\left(\sqrt{\tau^{\prime}+1}+\sqrt{\tau^{\prime}}\right)^{2}+\left(\sqrt{\tau^{\prime}+1}-\sqrt{\tau^{\prime}}\right)^{2}=2\left(\tau^{\prime}+1\right)+2 \tau^{\prime}=r .
$$

Let $u=w^{2}$, so that $r=\langle u\rangle$. Table 5 summarizes the relation between $\tau, r$ and $u$.

We can obtain an explicit relation between $v$ and $w$ as follows. Recall $v=\sqrt{\tau+1}+$ $\sqrt{\tau}$ and $w=\sqrt{\tau^{\prime}+1}+\sqrt{\tau^{\prime}}$. Changing the choice of square roots in these formulas would change $v$ or $w$ to another element in its orbit. To fix matters, select $\sqrt{\tau+1}, \sqrt{\tau}$, and $\sqrt{\tau^{\prime}}$ arbitrarily. By (36) $), \tau^{\prime}+1=1 /(\tau+1)$, so we may set $\sqrt{\tau^{\prime}+1}=1 / \sqrt{\tau+1}$. Let $i=\sqrt{\tau^{\prime}} \sqrt{\tau+1} / \sqrt{\tau}$. By (36),$i^{2}=\tau^{\prime}(\tau+1) / \tau=-1$. We have

$$
w=\sqrt{\tau^{\prime}+1}+\sqrt{\tau^{\prime}}=(1+i \sqrt{\tau}) / \sqrt{\tau+1} .
$$

Using that $\sqrt{\tau}=(v-1 / v) / 2$ and $\sqrt{\tau+1}=(v+1 / v) / 2$, we obtain (for a particular choice of $v, w$ within their orbits):

$$
w=\frac{2+i(v-1 / v)}{v+1 / v} .
$$

If $\tau$ and $\tau+1$ are nonzero squares, more can be said. In this case, Lemma 8.4 tells us that $r \in \mathcal{A}_{-2,2}^{\varepsilon,+}$ and $w^{q-\varepsilon}=1$. In particular, $r+2$ and $-(r-2)$ are nonzero squares. Let $\sqrt{r+2}$ and $\sqrt{2-r}$ denote any choice of square roots. Note that $u^{(q-\varepsilon) / 2}=w^{q-\varepsilon}=1$, and so $u^{(q-\varepsilon) / 4} \in\{1,-1\}$.

Proposition 8.5 Suppose that $\tau \in \mathcal{A}_{0,1}^{++}$, and let $r=\langle u\rangle \in \mathcal{A}_{-2,2}^{\varepsilon,+}$ be as in Table 5. As per Proposition 6.3, let $c$ be a square root of $1+\tau, c^{\prime}$ a square root of $1+1 / \tau$,

$$
\nu=\left(\frac{1 \pm 1 / c}{q}\right) \quad \text { and } \quad \mu=\left(\frac{1 \pm 1 / c^{\prime}}{q}\right) .
$$

Then

$$
\nu=\left(\frac{2}{q}\right) \mu, \quad \sqrt{r+2} \in \mathcal{A}_{-2,2}^{\varepsilon \mu, \mu}, \quad \sqrt{2-r} \in \mathcal{A}_{-2,2}^{\varepsilon \nu, \nu}, \quad u^{(q-\varepsilon) / 4}=\mu .
$$




\begin{tabular}{|c|c|c|}
\hline Condition on $\tau$ & Condition on $r$ & Condition on $u$ \\
\hline$\tau=0$ & $r=2$ & $u=1$ \\
\hline$\tau=\infty$ & $r=-2$ & $u=-1$ \\
\hline$\tau \in \mathcal{A}_{0,1}^{\varepsilon,+}$ & $r \in \mathcal{A}_{-2,2}^{++}$ & $u^{(q-1) / 2}=1, u^{2} \neq 1$ \\
\hline$\tau \in \mathcal{A}_{0,1}^{\varepsilon,-}$ & $r \in \mathcal{A}_{-2,2}^{--}$ & $u^{(q-1) / 2}=-1, u^{2} \neq 1$ \\
\hline$\tau \in \mathcal{A}_{0,1}^{-\varepsilon,+}$ & $r \in \mathcal{A}_{-2,2}^{-+}$ & $u^{(q+1) / 2}=1, u^{2} \neq 1$ \\
\hline$\tau \in \mathcal{A}_{0,1}^{-\varepsilon,-}$ & $r \in \mathcal{A}_{-2,2}^{+-}$ & $u^{(q+1) / 2}=-1, u^{2} \neq 1$ \\
\hline$\tau \in \mathcal{A}_{0,1}^{++}$ & $r \in \mathcal{A}_{-2,2}^{\varepsilon,+}$ & $u^{(q-\varepsilon) / 2}=1, u^{2} \neq 1$ \\
\hline$\tau \in \mathcal{A}_{0,1}^{+-}$ & $r \in \mathcal{A}_{-2,2}^{-\varepsilon,-}$ & $u^{(q-\varepsilon) / 2}=-1$ \\
\hline$\tau \in \mathcal{A}_{0,1}^{-+}$ & $r \in \mathcal{A}_{-2,2}^{-\varepsilon,+}$ & $u^{(q+\varepsilon) / 2}=1, u \neq 1$ \\
\hline$\tau \in \mathcal{A}_{0,1}^{--}$ & $r \in \mathcal{A}_{-2,2}^{\varepsilon,-}$ & $u^{(q+\varepsilon) / 2}=-1, u \neq-1$ \\
\hline
\end{tabular}

Table 5: Relation between $\tau, r$ and $u$ when $\tau \in \mathbb{F}_{q} \cup \infty, \tau \neq-1, \tau=(2-r) /(2+r)$, $r=\langle u\rangle$. (When $\tau \in\{0, \infty\}$, use (33), and when $\tau \in \mathbb{F}_{q} \backslash\{0,-1\}$, use Corollary 4.2.)

Proof. By Proposition 6.3, $\nu$ and $\mu$ are well defined and $\nu=\left(\frac{2}{q}\right) \mu$. Now $2 / c \in \mathcal{A}_{-2,2}^{\varepsilon \mu, \mu}$ because

$$
\begin{aligned}
& \left(\frac{2 / c+2}{q}\right)=\left(\frac{2(1+1 / c)}{q}\right)=\left(\frac{2}{q}\right) \nu=\mu, \\
& \left(\frac{2 / c-2}{q}\right)=\left(\frac{-1}{q}\right)\left(\frac{2}{q}\right)\left(\frac{1-1 / c}{q}\right)=\varepsilon \mu .
\end{aligned}
$$

The same reasoning applied to $-c$ shows that $-2 / c \in \mathcal{A}_{-2,2}^{\varepsilon \mu, \mu}$ as well. Since $(2 / c)^{2}=$ $4 /(\tau+1)=r+2$, this shows $\sqrt{r+2}= \pm 2 / c \in \mathcal{A}_{-2,2}^{\varepsilon \mu, \mu}$.

Now we show $u^{(q-\varepsilon) / 4}=\mu$. Write $r^{\prime}=\sqrt{r+2}=\left\langle s^{2}\right\rangle$, where $s \in \overline{\mathbb{F}}_{q}$. Since $r^{\prime} \in \mathcal{A}_{-2,2}^{\varepsilon \mu, \mu}$, we have $s^{q-\varepsilon}=\mu$ and $s^{4} \neq 1$ by Corollary 4.2. Now $r+2=\left(r^{\prime}\right)^{2}=\left\langle s^{2}\right\rangle^{2}=\left\langle s^{4}\right\rangle+2$, so $r=\left\langle s^{4}\right\rangle$. Since also $r=\langle u\rangle$, this shows $u=s^{4}$ or $u=s^{-4}$. Thus, $s^{q-\varepsilon}=\mu$ implies that $u^{(q-\varepsilon) / 4}=s^{ \pm(q-\varepsilon)}=\mu^{ \pm 1}$. Since $\mu=\mu^{-1}$, we conclude that $u^{(q-\varepsilon) / 4}=\mu$.

It remains only to prove that $\sqrt{2-r} \in \mathcal{A}_{-2,2}^{\varepsilon \nu, \nu}$. Let $\widetilde{\tau}=1 / \tau, \widetilde{r}=-r$, and $\widetilde{u}=-u$. Then $\widetilde{\tau} \in \mathcal{A}_{0,1}^{++}, \widetilde{\tau}=(2-\widetilde{r}) /(2+\widetilde{r})$ and $\widetilde{r}=\langle\widetilde{u}\rangle$. Note that replacing $\tau$ by $\widetilde{\tau}$ has the effect of exchanging $c$ with $c^{\prime}$ and $\nu$ with $\mu$. In particular, since we have already shown that $\sqrt{2+r} \in \mathcal{A}_{-2,2}^{\varepsilon \mu, \mu}$, it follows that $\sqrt{2-r}=\sqrt{2+\widetilde{r}} \in \mathcal{A}_{-2,2}^{\varepsilon \nu, \nu}$.

8.4. Deterministic square roots. Theorem 7.2 describes $\prod \mathcal{S}_{k, \ell}^{ \pm, \pm}$in terms of square roots of $\tau, \tau+1$, or $\tau /(\tau+1)$. In this section, we give a prescription for how this square root is unambiguously determined. As a word of warning, these square roots do not deserve to be called "canonical". For example, when $q=11$ and $\tau=1$, our deterministic square root of $\tau$ turns out to be -1 . 
As usual, we set $j=4 \tau /(\tau+1), \ell=4 /(\tau+1), r=\ell-2$. (See equations (32)-(37).). Assume $\tau \in \mathbb{F}_{q} \backslash\{0,-1\}$; then $j$ and $\ell$ are nonzero, and $r \notin\{2,-2\}$. Write $r=\langle u\rangle$; then $u=\left(r \pm \sqrt{r^{2}-4}\right) / 2$ may be explicitly computed in a quadratic extension. Since $r \notin\{2,-2\}$, we know $u^{2} \neq 1$. Any rational function of $u$ that is invariant under $u \mapsto 1 / u$ is a symmetric function of $u$ and $1 / u$, so may be expressed as a rational function of $u+1 / u$ and $u \cdot 1 / u=1$. In other words, it is a rational function of $u+1 / u=r$. Since $r=2(1-\tau) /(\tau+1)$, it is also a rational function of $\tau$. Our goal is to produce welldetermined square roots of $\tau, \tau+1$, or $\tau /(\tau+1)$ when exactly one of these quantities is a square in $\mathbb{F}_{q}$. We do this by finding a rational expression in $u$ whose square is $\tau$, $\tau+1$, or $\tau /(\tau+1)$, and that is invariant under $u \mapsto 1 / u$.

(i) Suppose that $\tau \in \mathcal{A}_{0,1}^{+-}$(equivalently, $j$ and $\ell$ are nonsquares). We will produce a deterministic square root of $\tau=j / \ell$. By Lemma 8.4 or Table 5, $r \in \mathcal{A}_{-2,2}^{-\varepsilon,-}$ and $u^{(q-\varepsilon) / 2}=-1$. Let

$$
a_{1}=\frac{u^{(q-\varepsilon) / 4}-u^{-(q-\varepsilon) / 4}}{u-1 / u} .
$$

This is invariant under $u \mapsto 1 / u$, and

$$
a_{1}^{2}=\frac{u^{(q-\varepsilon) / 2}+u^{-(q-\varepsilon) / 2}-2}{u^{2}+1 / u^{2}-2}=\frac{(-1)+(-1)-2}{(u+1 / u)^{2}-4}=\frac{-4}{r^{2}-4} .
$$

Thus, $\left(2 / a_{1}\right)^{2}=4-r^{2}=j \ell,\left(2 / a_{1} \ell\right)^{2}=j / \ell=\tau$. We define our square root by $\sqrt{\tau}=\sqrt{j / \ell}=2 /\left(a_{1} \ell\right)$.

(ii) Suppose that $\tau \in \mathcal{A}_{0,1}^{-+}$(equivalently, $j$ is a nonsquare and $\ell$ is a nonzero square). We will produce deterministic square roots of $\tau+1$ and $\ell$. By Lemma 8.4 or Table 5 , $r \in \mathcal{A}_{-2,2}^{-\varepsilon,+}$ and $u^{(q+\varepsilon) / 2}=1$. Let $a_{2}=\left\langle u^{(q-\varepsilon) / 4}\right\rangle$. Then $a_{2}^{2}=u^{(q-\varepsilon) / 2}+u^{-(q-\varepsilon) / 2}+2$. Now $u^{(q-\varepsilon) / 2}=u^{(q+\varepsilon) / 2-\varepsilon}=u^{-\varepsilon}$, so $a_{2}^{2}=u^{-\varepsilon}+u^{\varepsilon}+2=r+2=\ell$. We define $\sqrt{\ell}=a_{2}$. Since $\ell=4 /(\tau+1)$, we define $\sqrt{\tau+1}=2 / a_{2}=2 / \sqrt{\ell}$.

(iii) Suppose that $\tau \in \mathcal{A}_{0,1}^{--}$(equivalently, $j$ is a nonzero square and $\ell$ is a nonsquare). We will produce deterministic square roots of $\tau /(\tau+1)$ and $j$. By Lemma 8.4 or Table 5, $r \in \mathcal{A}_{-2,2}^{\varepsilon,-}$ and $u^{(q+\varepsilon) / 2}=-1$. Let $a_{3}=\left\langle(-u)^{(q-\varepsilon) / 4}\right\rangle=\left(\frac{2}{q}\right)\left\langle u^{(q-\varepsilon) / 4}\right\rangle$. Then $a_{3}^{2}=\left\langle u^{(q-\varepsilon) / 4}\right\rangle^{2}=u^{(q-\varepsilon) / 2}+u^{-(q-\varepsilon) / 2}+2$. Now $u^{(q-\varepsilon) / 2}=u^{(q+\varepsilon) / 2-\varepsilon}=-u^{-\varepsilon}$, so $a_{3}^{2}=-u^{-\varepsilon}-u^{\varepsilon}+2=-r+2=j$. We define $\sqrt{j}=a_{3}$ to be our deterministic square root. Since $j=4 \tau /(\tau+1)$, we define $\sqrt{\tau /(\tau+1)}=a_{3} / 2$.

Remark. Exchanging $j$ and $\ell$ is equivalent to $\tau \mapsto 1 / \tau, r \mapsto-r, u \mapsto-u$. Further, $\tau \in \mathcal{A}_{0,1}^{\varepsilon_{1}, \varepsilon_{2}} \Longleftrightarrow 1 / \tau \in \mathcal{A}_{0,1}^{\varepsilon_{1}, \varepsilon_{1} \varepsilon_{2}}$. Denote $\widetilde{\tau}=1 / \tau, \widetilde{r}=-r, \widetilde{u}=-u$. In $(i)$, if we set $\widetilde{a}_{1}=\left(\widetilde{u}^{m}+\widetilde{u}^{-m}\right) /(\widetilde{u}-1 / \widetilde{u})$ with $m=(q-\varepsilon) / 4$, then $\widetilde{a}_{1}=(-1)^{m+1} a_{1}=-\left(\frac{2}{q}\right) a_{1}$. Thus, $\sqrt{\widetilde{\tau}} \sqrt{\tau}=2 /\left(\widetilde{a}_{1} j\right) \times 2 /\left(a_{1} \ell\right)=-\left(\frac{2}{q}\right)\left(2 / a_{1}\right)^{2} /(j \ell)=-\left(\frac{2}{q}\right) . \quad$ In $(i i), \tau \in \mathcal{A}_{0,1}^{-+}$implies $\widetilde{\tau} \in \mathcal{A}_{0,1}^{--}$. The square root of $\ell$ is defined with respect to $\tau$ in $(i i)$ as $\sqrt{\ell}=a_{2}=\left\langle u^{m}\right\rangle$. On the other hand, $\sqrt{\ell}$ is defined with respect to $\widetilde{\tau}$ in $($ iii $)$ as $\sqrt{\ell}=\widetilde{a}_{3}$, where $\widetilde{a}_{3}=$ $\left\langle(-\widetilde{u})^{m}\right\rangle=\left\langle u^{m}\right\rangle=a_{2}$. These two definitions for $\sqrt{\ell}$ are consistent. 


\subsection{Proof of Theorem 7.1 (iv) and Theorem 7.2.}

We are in position to prove Theorems $7.1(i v)$ and 7.2. As usual, let $\tau \in \mathbb{F}_{q} \backslash\{0,-1\}$, and let $j, k, \ell, r, \tau^{\prime}$ be as in (32)-(37). Write $r=\left\langle w^{2}\right\rangle=\langle u\rangle$, where $w$ is as in Lemma 8.4 and $u=w^{2}$. The hypothesis that $\tau \neq 0, \infty$ implies $u^{2} \neq 1$.

Proof of Theorem 7.1 (iv): The hypothesis is that $\tau \in \mathcal{A}_{0,1}^{++}$. By Lemma 8.4, $r \in$ $\mathcal{A}_{-2,2}^{\varepsilon,+}$ and $u^{(q-\varepsilon) / 2}=w^{q-\varepsilon}=1$. By (4) and Proposition $8.3(i), \prod \mathcal{T}_{j, \ell}^{--}=\prod \mathcal{S}_{k, \ell}^{-\varepsilon,-}=$ $\prod \mathcal{S}_{r-2, r+2}^{-\varepsilon,-}=\left(\frac{2}{q}\right)\left\langle u^{(q-\varepsilon) / 4}\right\rangle$. By Proposition 8.5 and $(\underline{34}), u^{(q-\varepsilon) / 4}=\left(\frac{2 \pm 2 / \sqrt{\tau+1}}{q}\right)=$ $\left(\frac{2 \pm \sqrt{\ell}}{q}\right)$. Thus,

$$
\prod \mathcal{T}_{j, \ell}^{--}=\left(\frac{2}{q}\right)\left\langle u^{(q-\varepsilon) / 4}\right\rangle=\left(\frac{2}{q}\right)\left(\frac{2 \pm \sqrt{\ell}}{q}\right) \cdot 2=\left(\frac{2 \pm \sqrt{j}}{q}\right) \cdot 2
$$

where in the last step we use (30). By Proposition [5.2, $\prod \mathcal{T}_{\ell, j}^{--}=\left(\frac{2}{q}\right) \prod \mathcal{T}_{j, \ell}^{--}$. Proof of Theorem 7.2: We continue with the above notation.

(i) The hypothesis is that $\tau \in \mathcal{A}_{0,1}^{+-}$. By Lemma 8.4 or Table $5, r \in \mathcal{A}_{-2,2}^{-\varepsilon,-}$. Then, by Proposition 8.3) $(i i)$,

$$
\prod \mathcal{S}_{k, \ell}^{\varepsilon,+}=\prod \mathcal{S}_{r-2, r+2}^{\varepsilon,+}=-\left(\frac{2}{q}\right)\left(u^{(q-\varepsilon) / 4}-u^{-(q-\varepsilon) / 4}\right) /(u-1 / u)=-\left(\frac{2}{q}\right) a_{1}
$$

where $a_{1}$ is as in Section 8.4. The deterministic square root of $\tau$ is defined by $\sqrt{\tau}=$ $2 /\left(a_{1} \ell\right)$. Thus, $a_{1}=2 /(\sqrt{\tau} \ell)=2 /(\ell \sqrt{j / \ell})$, and the result follows.

(ii) The hypothesis is that $\tau \in \mathcal{A}_{0,1}^{-+}$. By Lemma 8.4 or Table $5, r \in \mathcal{A}_{-2,2}^{-\varepsilon,+}$. By Proposition $8.3(i), \prod \mathcal{S}_{r-2, r+2}^{-\varepsilon,-}=\left(\frac{2}{q}\right)\left\langle u^{(q-\varepsilon) / 4}\right\rangle$. This is equal to $\left(\frac{2}{q}\right) a_{2}$, where $a_{2}=$ $\sqrt{\ell}=2 / \sqrt{\tau+1}$ is as in Section 8.4. The result follows.

(iii) We are assuming $\tau \in \mathcal{A}_{0,1}^{--}$. By Lemma 8.4 or Table 5, $r \in \mathcal{A}_{-2,2}^{\varepsilon,-}$ and $u^{(q+\varepsilon) / 2}=-1$. By Proposition $\underline{8.3}(i), \prod \mathcal{S}_{k, \ell}^{-\varepsilon,-}=\prod \mathcal{S}_{r-2, r+2}^{-\varepsilon,-}=\left(\frac{2}{q}\right)\left\langle u^{(q-\varepsilon) / 4}\right\rangle$. This equals $a_{3}=\sqrt{j}=$ $2 \sqrt{\tau /(\tau+1)}$, where $a_{3}$ is defined in Section 8.4 .

\section{Rational reciprocity}

It sometimes happens that more than one row of Table 2 applies for a given value of $\tau$. It turns out that one can obtain some known results about rational reciprocity in this way. An excellent reference for reciprocity laws, including their historical development, is by Lemmermeyer, [4].

For example, if $\tau=1$ then $\tau$ and $\tau+1$ are squares iff $\left(\frac{2}{q}\right)=1$, or equivalently, iff $q \equiv \pm 1(\bmod 8)$. Assuming this, then the third row of Table 2 tells us that

$$
\prod \mathcal{T}_{2,2}^{-+}=(-1)^{\lfloor(q+3) / 8\rfloor} .
$$

On the other hand, the two bottom rows of Table 2 tell us that $\prod \mathcal{T}_{2,2}^{-+}=\left(\frac{1 \pm 1 / \sqrt{2}}{q}\right)$. 
On multiplying the right side by $\left(\frac{2}{q}\right)=1$, we obtain

$$
\prod \mathcal{T}_{2,2}^{-+}=\left(\frac{2 \pm \sqrt{2}}{q}\right)
$$

On comparison of (43) and (44), we find

$$
\left(\frac{2 \pm \sqrt{2}}{q}\right)=\left\{\begin{array}{lll}
(-1)^{(q-1) / 8} & \text { if } q \equiv 1 & (\bmod 8) \\
(-1)^{(q+1) / 8} & \text { if } q \equiv 7 & (\bmod 8)
\end{array}\right.
$$

A different proof of this result can be found in [4, p. 166].

Another interesting phenomenon is the following theorem and corollary.

Proposition 9.1 Suppose that $\left(\frac{2}{q}\right)=1$ (equivalently, $q \equiv \varepsilon(\bmod 8)$ where $\varepsilon \in$ $\{1,-1\})$, and let $\sqrt{2} \in \mathbb{F}_{q}$ denote a (fixed) square root of 2. If $q \equiv \varepsilon(\bmod 16)$ then

$$
\prod \mathcal{T}_{2-\sqrt{2}, 2+\sqrt{2}}^{--}=\prod\left\{a \in \mathbb{F}_{q}^{\times}: 2 \pm(\sqrt{2}+a) \text { are nonsquares }\right\}=(-1)^{(q-\varepsilon) / 16} \cdot 2 .
$$

If $q \equiv 8+\varepsilon(\bmod 16)$, then

$\prod \mathcal{T}_{2-\sqrt{2}, 2+\sqrt{2}}^{++}=\prod\left\{a \in \mathbb{F}_{q}^{\times}: 2 \pm(\sqrt{2}+a)\right.$ are nonzero squares $\}=(-1)^{(q+8-\varepsilon) / 16} \cdot \sqrt{2}$.

Proof. Let $\zeta$ be a primitive eighth root of unity in $\overline{\mathbb{F}}_{q}$, and let $r=\langle\zeta\rangle$. Now $\zeta^{q-\varepsilon}=1$, so $r^{q}=\left\langle\zeta^{q}\right\rangle=\left\langle\zeta^{\varepsilon}\right\rangle=\langle\zeta\rangle=r$. Further, since $\zeta^{2}=i$ is a square root of -1 , we have $r^{2}=\zeta^{2}+\zeta^{-2}+2=i+1 / i+2=2$; therefore $r= \pm \sqrt{2}$. By changing $\zeta$ to $-\zeta$ if necessary, we can assume $r=\sqrt{2}=\langle\zeta\rangle$.

If $q \equiv \varepsilon(\bmod 16)$ then by (45), $2 \pm \sqrt{2}$ are squares, so $\sqrt{2} \notin \mathcal{A}_{-2,2}^{-\varepsilon,-}$. By Proposition $8.3(i)$ and (4),$\prod \mathcal{T}_{2-\sqrt{2}, 2+\sqrt{2}}^{--}=\left\langle(-\zeta)^{(q-\varepsilon) / 4}\right\rangle$. Writing $q=\varepsilon+16 \mu$ where $\mu \in \mathbb{Z}$, the right side is $\left\langle(-\zeta)^{4 \mu}\right\rangle=(-1)^{\mu} \cdot 2$. This proves the first formula.

Next, if $q \equiv 8+\varepsilon(\bmod 16)$ then by (45) $), 2 \pm \sqrt{2}$ are nonsquares, so $\sqrt{2} \notin \mathcal{A}_{-2,2}^{\varepsilon,+}$. By Proposition 8.3 ( $i i)$ and (44),

$$
\prod \mathcal{T}_{2-\sqrt{2}, 2+\sqrt{2}}^{++}=-\left((-\zeta)^{(q-\varepsilon) / 4}-(-\zeta)^{-(q-\varepsilon) / 4}\right) /(\zeta-1 / \zeta)
$$

Writing $q=8+\varepsilon+16 \mu$, the numerator is

$$
(-1)\left((-\zeta)^{2+4 \mu}-(-\zeta)^{-(2+4 \mu)}\right)
$$

Since $\zeta^{4}=-1$, this equals $(-1)^{\mu+1}\left(\zeta^{2}-\zeta^{-2}\right)$. Thus, $\prod \mathcal{T}_{2-\sqrt{2}, 2+\sqrt{2}}^{++}=(-1)^{\mu+1}\left(\zeta^{2}-\right.$ $\left.\zeta^{-2}\right) /(\zeta-1 / \zeta)=(-1)^{\mu+1}\langle\zeta\rangle=(-1)^{\mu+1} \sqrt{2}=(-1)^{(q+8-\varepsilon) / 16} \sqrt{2}$.

Corollary 9.2 Suppose that $q \equiv \varepsilon(\bmod 16)$, so that $2+\sqrt{2}$ is a square in $\mathbb{F}_{q}$ (for either choice of square root) by (45). Then $2+\sqrt{2+\sqrt{2}}$ and $2-\sqrt{2+\sqrt{2}}$ are both squares if $q \equiv \varepsilon(\bmod 32)$, and otherwise they are both nonsquares. 
Proof. They are both squares or both nonsquares because the product, $4-(2+$ $\sqrt{2})=2-\sqrt{2}$, is a square. In Table 2, set $j=2-\sqrt{2}$ and $\ell=2+\sqrt{2}$. Then $\tau=(2-\sqrt{2}) /(2+\sqrt{2}), \tau+1=4 /(2+\sqrt{2}), 2+2 / \sqrt{\tau+1}=2+\sqrt{2+\sqrt{2}}$. According to the table, $\prod \mathcal{T}_{j, \ell}^{--}=2$ if $2+2 / \sqrt{\tau+1}$ is a square, and -2 otherwise. On the other hand, Proposition 9.1 asserts that $\prod \mathcal{T}_{j, \ell}^{--}=(-1)^{(q-\varepsilon) / 16} \cdot 2$. The result follows.

One can continue down this path indefinitely, although the easiest proof does not involve Dickson polynomials. The next lemma is well known.

Lemma 9.3 If $u \in \overline{\mathbb{F}}_{q}$ is a primitive $d$-th root of unity, where $(d, q)=1$, then $\langle u\rangle \in$ $\mathbb{F}_{q} \Longleftrightarrow q \equiv \pm 1(\bmod d)$.

Proof. In general, $\langle u\rangle=\langle v\rangle$ iff $v \in\{u, 1 / u\}$. Thus, $\langle u\rangle \in \mathbb{F}_{q} \Longleftrightarrow\left\langle u^{q}\right\rangle=\langle u\rangle \Longleftrightarrow$ $u^{q} \in\{u, 1 / u\}$. The latter is equivalent to $u^{q-1}=1$ or $u^{q+1}=1$, i.e., $d \mid q-1$ or $d \mid q+1$. Thus, $\langle u\rangle \in \mathbb{F}_{q} \Longleftrightarrow q \equiv \pm 1(\bmod d)$.

Proposition 9.4 In $\overline{\mathbb{F}}_{q}$, let $b_{0}=\sqrt{2}, b_{1}=\sqrt{2+b_{0}}, b_{2}=\sqrt{2+b_{1}}$, and in general, $b_{i}=\sqrt{2+b_{i-1}}$, where the choice of square root is arbitrary at each stage. Then $b_{i} \in \mathbb{F}_{q}$ iff $q \equiv \varepsilon\left(\bmod 2^{i+3}\right)$, where $\varepsilon=\left(\frac{-1}{q}\right) \in\{1,-1\}$.

Proof. First, we show by induction that $b_{j}=\left\langle\zeta_{j+3}\right\rangle$, where $\zeta_{j}$ is a primitive $2^{j}$ root of unity 1 in $\mathbb{F}_{q}$. To start the induction, $\sqrt{2}=\left\langle\zeta_{3}\right\rangle$ was demonstrated in the proof of Proposition 9.1. Now assume the induction hypothesis for $j$ and we prove it for $j+1$. Let $\zeta_{j+4}^{2}=\zeta_{j+3}$; then $\zeta_{j+4}$ is a primitive $2^{j+4}$ root of unity. Now $\left\langle\zeta_{j+4}\right\rangle^{2}=\left\langle\zeta_{j+3}\right\rangle+2=b_{j}+2$, so $\left\langle\zeta_{j+4}\right\rangle= \pm \sqrt{b_{j}+2}= \pm b_{j+1}$. Replacing $\zeta_{j+4}$ by its negative if needed, we may assume that $\left\langle\zeta_{j+4}\right\rangle=b_{j+1}$. Finally, $b_{j} \in \mathbb{F}_{q} \Longleftrightarrow\left\langle\zeta_{j+3}\right\rangle \in \mathbb{F}_{q} \Longleftrightarrow q \equiv \pm 1\left(\bmod 2^{j+3}\right)$ by Lemma 9.3 .

The above results generalize in various ways.

Proposition 9.5 In $\overline{\mathbb{F}}_{q}$, suppose $u_{0}$ is a primitive $2 k$-th root of unity and $b_{0}=\left\langle u_{0}\right\rangle$. Let $b_{1}=\sqrt{2+b_{0}}, \ldots, b_{i}=\sqrt{2+b_{i-1}}, \ldots$, for any choice of square roots. Then

(i) There are $u_{1}, u_{2}, \ldots \in \overline{\mathbb{F}}_{q}$ such that $u_{i}^{2}=u_{i-1}$ and $b_{i}=\left\langle u_{i}\right\rangle$.

(ii) For $i \geq 0, b_{i} \in \mathbb{F}_{q}$ iff $q \equiv \pm 1\left(\bmod 2^{i+1} k\right)$.

(iii) Suppose $q \equiv \varepsilon(\bmod 4 k)$, where as usual $\varepsilon=\left(\frac{-1}{q}\right)$, and write $q=\varepsilon+4 k \mu$. Then

$$
\prod \mathcal{T}_{2-b_{0}, 2+b_{0}}^{--}=\prod\left\{a \in \mathbb{F}_{q}^{\times}: 2 \pm\left(b_{0}+a\right) \text { are nonsquares }\right\}=(-1)^{(k+1) \mu} \cdot 2 .
$$

Proof. Let $u_{1}^{2}=u_{0}$. Then $\left\langle u_{1}\right\rangle^{2}=u_{0}+u_{0}^{-1}+2=b_{0}+2$, so $b_{1}= \pm\left\langle u_{1}\right\rangle$. Replacing $u_{1}$ by $-u_{1}$ if necessary, we can assume $b_{1}=\left\langle u_{1}\right\rangle$. By induction, we can find $u_{2}, u_{3}, \ldots$ such that $u_{i}^{2}=u_{i-1}$ and $b_{i}=\left\langle u_{i}\right\rangle$. Clearly $u_{i}$ is primitive of order $2^{i}(2 k)$. By Lemma 9.3, $b_{i} \in \mathbb{F}_{q} \Longleftrightarrow q \equiv \pm 1\left(\bmod 2^{i}(2 k)\right)$. This proves $(i)$ and $(i i)$. Now we prove (iii). The hypothesis $q \equiv \varepsilon(\bmod 4 k)$ implies $b_{1} \in \mathbb{F}_{q}$ by $(i i)$, therefore $2+b_{0}$ is a square. In particular, $b_{0} \notin \mathcal{A}_{-2,2}^{-\varepsilon,-}$. By Proposition $8.3(i)$ and (4), $\prod \mathcal{T}_{2-b_{0}, 2+b_{0}}^{--}=\left\langle\left(-u_{0}\right)^{k \mu}\right\rangle$. Since $u_{0}^{k}=-1$, we have $\left(-u_{0}\right)^{k \mu}=(-1)^{k \mu}(-1)^{\mu}=(-1)^{(k+1) \mu}$. The result follows.

\footnotetext{
${ }^{1}$ Here the $2^{j+1}$ choices of square roots in the definition of $b_{j}$ correspond to the $2^{j+2}$ distinct choices for primitive $2^{j+3}$ roots of unity, modulo the relation $\langle\zeta\rangle=\left\langle\zeta^{-1}\right\rangle$.
} 
Proposition 9.6 (i) Suppose $(q, 6)=1$. In $\overline{\mathbb{F}}_{q}$, let $b_{0}=\sqrt{3}, b_{1}=\sqrt{2+b_{0}}, \ldots, b_{i}=$ $\sqrt{2+b_{i-1}}, \ldots$, for any choice of square roots. Then $b_{i} \in \mathbb{F}_{q}$ iff $q \equiv \pm 1\left(\bmod 12 \cdot 2^{i}\right)$. (ii) Suppose $(q, 10)=1$. In $\overline{\mathbb{F}}_{q}$, let $b_{0}=(1-\sqrt{5}) / 2, b_{1}=\sqrt{2+b_{0}}, \ldots, b_{i}=\sqrt{2+b_{i-1}}, \ldots$, for any choice of square roots. Then $b_{i} \in \mathbb{F}_{q}$ iff $q \equiv \pm 1\left(\bmod 10 \cdot 2^{i}\right)$.

Proof. Using Proposition 9.5, it suffices to show that

$$
\sqrt{3}=\left\langle\zeta_{12}\right\rangle \text { and }(1-\sqrt{5}) / 2=\left\langle\zeta_{10}\right\rangle
$$

where $\zeta_{j}$ denotes a primitive $j$-th root of unity.

To prove this for $\sqrt{3}$, let $\omega=-\zeta_{12}^{2}$; then $\omega^{3}=-\zeta_{12}^{6}=1$. Noting that $\omega^{2}+\omega+1=0$, we have $\langle\omega\rangle=-1$. Then $\left\langle\zeta_{12}\right\rangle^{2}=\left\langle\zeta_{12}^{2}\right\rangle+2=\langle-\omega\rangle+2=-\langle\omega\rangle+2=3$. Thus, $\sqrt{3}= \pm\left\langle\zeta_{12}\right\rangle=\left\langle \pm \zeta_{12}\right\rangle$. Replacing $\zeta_{12}$ by $-\zeta_{12}$ if necessary, we can assume $\sqrt{3}=\left\langle\zeta_{12}\right\rangle$.

To prove this for $(1-\sqrt{5}) / 2$, let $\zeta$ be a primitive fifth root of unity. Now $\langle\zeta\rangle+\left\langle\zeta^{2}\right\rangle=$ $\zeta+\zeta^{4}+\zeta^{2}+\zeta^{3}=-1$, so $(\langle\zeta\rangle+1 / 2)^{2}=\langle\zeta\rangle^{2}+\langle\zeta\rangle+1 / 4=\left(\left\langle\zeta^{2}\right\rangle+2\right)+\langle\zeta\rangle+1 / 4=2-1+$ $1 / 4=5 / 4$. This shows that $2\langle\zeta\rangle+1$ is a square root of 5 . The other square root of 5 is $2\left\langle\zeta^{2}\right\rangle+1=-(2\langle\zeta\rangle+1)$. Replacing $\zeta$ by $\zeta^{2}$ if necessary gives $\langle-\zeta\rangle=-\langle\zeta\rangle=(1-\sqrt{5}) / 2$. Set $\zeta_{10}=-\zeta$, which is a primitive tenth root of unity.

We remark that Lemma 9.3 , together with the formulas

$$
\left\langle\zeta_{8}\right\rangle=\sqrt{2}, \quad\left\langle\zeta_{12}\right\rangle=\sqrt{3}, \quad \text { and } \quad\left\langle\zeta_{10}\right\rangle=(1-\sqrt{5}) / 2,
$$

give one-line proofs:

$$
\begin{gathered}
\left(\frac{2}{q}\right)=1 \Longleftrightarrow \sqrt{2} \in \mathbb{F}_{q} \Longleftrightarrow\left\langle\zeta_{8}\right\rangle \in \mathbb{F}_{q} \Longleftrightarrow q \equiv \pm 1 \quad(\bmod 8), \\
\left(\frac{3}{q}\right)=1 \Longleftrightarrow \sqrt{3} \in \mathbb{F}_{q}^{\times} \Longleftrightarrow 3 \nmid q \text { and }\left\langle\zeta_{12}\right\rangle \in \mathbb{F}_{q} \Longleftrightarrow q \equiv \pm 1 \quad(\bmod 12) . \\
\left(\frac{5}{q}\right)=1 \Longleftrightarrow \sqrt{5} \in \mathbb{F}_{q}^{\times} \Longleftrightarrow 5 \nmid q \text { and }\left\langle\zeta_{10}\right\rangle \in \mathbb{F}_{q} \Longleftrightarrow q \equiv \pm 1 \quad(\bmod 10) .
\end{gathered}
$$

Proposition 9.7 Suppose that $\left(\frac{3}{q}\right)=1$ (equivalently, $q \equiv \pm 1(\bmod 12)$ ), and let $\sqrt{3} \in \mathbb{F}_{q}$ denote a (fixed) square root of 3. Let $\nu=(-1)^{(q-\varepsilon) / 12}$, i.e., $\nu=1$ if $q \equiv \pm 1$ $(\bmod 24), \nu=-1$ otherwise. Then

$$
\prod \mathcal{T}_{2-\sqrt{3}, 2+\sqrt{3}}^{-\nu,-\nu}=(-1)^{\mu} \cdot 2, \quad \text { where } \mu=\lfloor(q+1) / 24\rfloor
$$

Proof. Write $\sqrt{3}=\langle u\rangle$, where $u=\zeta_{12}$ is a primitive twelfth root of unity. By Proposition 9.5 (iii), if $q \equiv \pm 1(\bmod 24)$ then $\prod \mathcal{T}_{2-\sqrt{3}, 2+\sqrt{3}}^{--}=(-1)^{\mu} \cdot 2$.

Now suppose $q \not \equiv \pm 1(\bmod 24)$. Then we may write $q=\varepsilon+12+24 \mu$, where $\varepsilon \in\{1,-1\}$. By Proposition $9.5(i i), \sqrt{2+\sqrt{3}} \notin \mathbb{F}_{q}, \quad$ i.e., $\left(\frac{2+\sqrt{3}}{q}\right)=-1$. Then $\sqrt{3} \notin \mathcal{A}_{-2,2}^{\varepsilon,+}$. By Proposition 8.3 (ii) and (44),

$$
\prod \mathcal{T}_{2-\sqrt{3}, 2+\sqrt{3}}^{++}=-\left((-u)^{(q-\varepsilon) / 4}-(-u)^{-(q-\varepsilon) / 4}\right) /(u-1 / u) .
$$


Since $u^{6}=-1$, the numerator of the right side is $(-1)\left((-u)^{3+6 \mu}-(-u)^{-(3+6 \mu)}\right)=$ $u^{3+6 \mu}-u^{-3-6 \mu}=(-1)^{\mu}\left(u^{3}-u^{-3}\right)$. Then the right side is $(-1)^{\mu}\left(u^{3}-u^{-3}\right) /(u-1 / u)=$ $(-1)^{\mu}\left(u^{2}+1+u^{-2}\right)$. Let $\omega=-u^{2}$; then $\omega^{3}=-u^{6}=1$, so $\omega^{2}+\omega+1=0$. We have $u^{2}+1+u^{-2}=\langle-\omega\rangle+1=2$, so $\prod \mathcal{T}_{2-\sqrt{3}, 2+\sqrt{3}}^{++}=(-1)^{\mu} \cdot 2$.

Proposition 9.8 Suppose that $\left(\frac{5}{q}\right)=1$ (equivalently, $q \equiv \pm 1(\bmod 5)$ ), and let $r=$ $(1-\sqrt{5}) / 2$, where $\sqrt{5} \in \mathbb{F}_{q}$ denotes a (fixed) square root of 5 . Then

$$
\begin{array}{ll}
\prod \mathcal{T}_{2-r, 2+r}^{--}=2 & \text { if } q \equiv \pm 1 \quad(\bmod 20), \\
\prod \mathcal{T}_{2-r, 2+r}^{++}=-\varepsilon r & \text { otherwise. }
\end{array}
$$

Proof. Write $r=\langle u\rangle$, where $u=\zeta_{10}$ is a primitive tenth root of unity. If $q \equiv \pm 1$ (mod 20) then applying Proposition 9.5)(iii) with $k=5$ gives $\prod \mathcal{T}_{2-r, 2+r}^{--}=2$. Now suppose that $q \not \equiv \pm 1(\bmod 20)$. Being that $q$ is odd and $q \equiv \pm 1$ ( $\bmod 5)$ by hypothesis, we have $q \equiv \pm 1(\bmod 10)$. Write $q=10-\varepsilon+20 \mu$, where $\varepsilon \in\{1,-1\}$. Note that $q \equiv \varepsilon(\bmod 4)$, so $\varepsilon=\left(\frac{-1}{q}\right)$. By Proposition $9.5(i i)$ with $i=1,2+r$ is a nonsquare, so $r \notin \mathcal{A}_{-2,2}^{\varepsilon,+}$. By Proposition $8.3(i i)$,

$$
\prod \mathcal{T}_{2-r, 2+r}^{++}=-\left((-u)^{(q-\varepsilon) / 4}-(-u)^{-(q-\varepsilon) / 4}\right) /(u-1 / u) .
$$

We have $(q-\varepsilon) / 4=(10-2 \varepsilon+20 \mu) / 4=2+5 \mu+(1-\varepsilon) / 2$. Let $\zeta=-u$, so $\zeta^{5}=-u^{5}=1$. Then $(-u)^{(q-\varepsilon) / 4}=\zeta^{2+5 \mu+(1-\varepsilon) / 2}$. This is $\zeta^{2}$ if $\varepsilon=1$, or $\zeta^{3}=\zeta^{-2}$ if $\varepsilon=-1$. Therefore,

$$
\prod \mathcal{T}_{2-r, 2+r}^{++}= \begin{cases}\left(\zeta^{2}-\zeta^{-2}\right) /\left(\zeta-\zeta^{-1}\right) & \text { if } \varepsilon=1 \\ \left(\zeta^{-2}-\zeta^{2}\right) /\left(\zeta-\zeta^{-1}\right) & \text { if } \varepsilon=-1\end{cases}
$$

Thus, $\prod \mathcal{T}_{2-r, 2+r}^{++}=\varepsilon\langle\zeta\rangle=-\varepsilon\langle u\rangle=-\varepsilon r$.

\section{References}

[1] Antonia W. Bluher, Permutation properties of Dickson polynomials and connections to number theory, Finite Fields Appl. 76 (2021), 101899. Earlier version in arXiv:1707.06877 [math.NT], 21 July 2017.

[2] Antonia Bluher, A structure theorem for finite fields, Finite Fields Appl. 68 (2020), 101732 .

[3] Ivan Bjerre Damgard, On the randomness of Legendre and Jacobi sequences. In Shafi Goldwasser, editor, Advances in Cryptography - Crypto '88 (Lecture Notes in Computer Science 403), Springer-Verlag, 1990, pp. 163-172.

[4] Franz Lemmermeyer, Reciprocity Laws: from Euler to Eisenstein, Springer-Verlag, Berlin, Heidelberg, New York, 1990.

[5] R. Lidl, G.L. Mullen, G. Turnwald, Dickson Polynomials, Pitman Monographs in Pure and Applied Mathematics, Vol. 65, Longman Scientific \& Technical/John Wiley \& Sons, New York, 1993. 
[6] Rudolf Lidl and Harald Niederreiter, Finite Fields, Gian-Carlo Rota, Editor, Encyclopedia of Mathematics and Its Applications Vol. 20, Addison-Wesley Publishing Company, 1983.

[7] Oystein Ore, Number Theory and its History, McGraw-Hill, New York, 1948. Republished by Dover Books, New York, 1988.

[8] Ivan Niven, Herbert S. Zuckerman, and Hugh L. Montgomery, An Introduction to the Theory of Numbers - Fifth Edition, John Wiley \& Sons, 1991. 